\title{
Event-Driven Network Programming
}

\author{
Jedidiah McClurg \\ CU Boulder, USA \\ jedidiah.mcclurg@colorado.edu
}

\author{
Hossein Hojjat \\ Cornell University, USA \\ hojjat@cornell.edu
}

\author{
Nate Foster \\ Cornell University, USA \\ jnfoster@cs.cornell.edu
}

\author{
Pavol Černý \\ CU Boulder, USA \\ pavol.cerny@colorado.edu
}

\begin{abstract}
Software-defined networking (SDN) programs must simultaneously describe static forwarding behavior and dynamic updates in response to events. Event-driven updates are critical to get right, but difficult to implement correctly due to the high degree of concurrency in networks. Existing SDN platforms offer weak guarantees that can break application invariants, leading to problems such as dropped packets, degraded performance, security violations, etc. This paper introduces event-driven consistent updates that are guaranteed to preserve well-defined behaviors when transitioning between configurations in response to events. We propose network event structures (NESs) to model constraints on updates, such as which events can be enabled simultaneously and causal dependencies between events. We define an extension of the NetKAT language with mutable state, give semantics to stateful programs using NESs, and discuss provably-correct strategies for implementing NESs in SDNs. Finally, we evaluate our approach empirically, demonstrating that it gives well-defined consistency guarantees while avoiding expensive synchronization and packet buffering.
\end{abstract}

Categories and Subject Descriptors C.2.3 [Computercommunication Networks]: Network Operations-Network Management; D.3.2 [Programming Languages]: Language Classifications-Specialized application languages; D.3.4 [Programming Languages]: Processors-Compilers

Keywords network update, consistent update, event structure, software-defined networking, SDN, NetKAT

\section{Introduction}

Software-defined networking (SDN) allows network behavior to be specified using logically-centralized programs that

Permission to make digital or hard copies of all or part of this work for personal or classroom use is granted without fee provided that copies are not made or distributed for profit or commercial advantage and that copies bear this notice and the full citation on the first page. Copyrights for components of this work owned by others than ACM must be honored. Abstracting with credit is permitted. To copy otherwise, or republish, to post on servers or to redistribute to lists, requires prior specific permission and/or a fee. Request permissions from Permissions@acm.org.

Copyright is held by the owner/author(s). Publication rights licensed to ACM.

PLDI'16, June 13-17, 2016, Santa Barbara, CA, USA

ACM. 978-1-4503-4261-2/16/06 ..\$15.00

http://dx.doi.org/10.1145/2908080.2908097 execute on general-purpose machines. These programs react to events such as topology changes, traffic statistics, receipt of packets, etc. by modifying sets of forwarding rules installed on switches. SDN programs can implement a wide range of advanced network functionality including fine-grained access control [8], network virtualization [22], traffic engineering $[15,16]$, and many others.

Although the basic SDN model is simple, building sophisticated applications is challenging in practice. Programmers must keep track of numerous low-level details such as encoding configurations into prioritized forwarding rules, processing concurrent events, managing asynchronous events, dealing with unexpected failures, etc. To address these challenges, a number of domain-specific network programming languages have been proposed [2, 10, 19, 21, 29, $31,36,37]$. The details of these languages vary, but they all offer higher-level abstractions for specifying behavior (e.g., using mathematical functions, boolean predicates, relational operators, etc.), and rely on a compiler and run-time system to generate and manage the underlying network state.

Unfortunately, the languages that have been proposed so far lack critical features that are needed to implement dynamic, event-driven applications. Static languages such as NetKAT [2] offer rich constructs for describing network configurations, but lack features for responding to events and maintaining internal state. Instead, programmers must write a stateful program in a general-purpose language that generates a stream of NetKAT programs. Dynamic languages such as FlowLog and Kinetic [21, 31] offer stateful programming models, but they do not specify how the network behaves while it is being reconfigured in response to state changes. Abstractions such as consistent updates provide strong guarantees during periods of reconfiguration [26, 33], but current realizations are limited to properties involving a single packet (or set of related packets, such as a unidirectional flow). To implement correct dynamic SDN applications today, the most effective option is often to use low-level APIs, forgoing the benefits of higher-level languages entirely.

Example: Stateful Firewall. To illustrate the challenges that arise when implementing dynamic applications, consider a topology where an internal host $H_{1}$ is connected to switch $s_{1}$, an external host $H_{4}$ is connected to a switch $s_{4}$, and switches $s_{1}$ and $s_{4}$ are connected to each other (see Fig- 


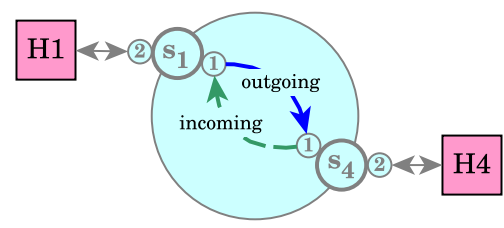

Figure 1: Topology for simple Stateful Firewall.

ure 1). Suppose we wish to implement a stateful firewall: at all times, host $H_{1}$ is allowed to send packets to host $H_{4}$, but $H_{4}$ should only be allowed to send packets to $H_{1}$ if $H_{1}$ previously initiated a connection. Implementing even this simple application turns out to be difficult, because it involves coordinating behavior across multiple devices and packets. The basic idea is that upon receiving a packet from $H_{1}$ at $s_{4}$, the program will need to issue a command to install a forwarding rule on $s_{4}$ allowing traffic to flow from $H_{4}$ back to $H_{1}$. There are two straightforward (but incorrect) implementation strategies on current SDN controllers.

1. The outgoing request from $H_{1}$ is diverted to the controller, which sets up flow tables for the incoming path and also forwards the packet(s) to $H_{4}$. Reconfiguring flow tables takes time, so $H_{4}$ 's response will likely be processed by the default drop rule. Even worse, if the response is the SYN-ACK in a TCP handshake, normal retransmission mechanisms will not help-the client will have to wait for a timeout and initiate another TCP connection. In practice, this greatly increases the latency of setting up a connection, and potentially wreaks havoc on application performance.

2. The outgoing request is buffered at the controller, which sets up the flow tables for the incoming path but waits until the rules are installed before forwarding the packet(s). This avoids the problem in (1), but places extra load on the controller and also implements the firewall incorrectly, since incoming traffic is allowed before the outgoing request is delivered. Leaving the network unprotected (even briefly) can be exploited by a malicious attacker.

Thus, while it is tempting to think that reliability mechanisms built into protocols such as TCP already prevent (or at least reduce) these types of errors, this is not the case. While it is true that some applications can tolerate long latencies, dropped packets, and weak consistency, problems with updates do lead to serious problems in practice. As another example, consider an intrusion detection system that monitors suspicious traffic-inadvertently dropping or allowing even a few packets due to a reconfiguration would weaken the protection it provides. The root of these problems is that existing SDN frameworks do not provide strong guarantees during periods of transition between configurations in response to events. An eventual guarantee is not strong enough to implement the stateful firewall correctly, and even a consistent update [33] would not suffice, since consistent updates only dictate what must happen to individual packets.
Existing Approaches. Experienced network operators may be able to use existing tools/methods to correctly implement event-driven configuration changes. However, as seen above, this requires thinking carefully about the potential interleavings of events and updates, delegating atomic operations to the controller (incurring a performance hit), etc.

As mentioned, there are stateful programming systems that attempt to make this process easier for the programmer, but update strategies in these systems either offer no consistency guarantees during dynamic updates, rely on expensive processing via the controller, and/or require the programmer to craft an update protocol by hand. In this paper, we group these approaches together, using the term uncoordinated update to describe their lack of support for coordinating local updates in a way that ensures global consistency.

Event-Driven Consistent Update. We propose a new semantic correctness condition with clear guarantees about updates triggered by events. This enables specification of how the network should behave during updates, and enables precise formal reasoning about stateful network programs.

An event-driven consistent update is denoted as a triple $C_{i} \stackrel{e}{\rightarrow} C_{f}$, where $C_{i}$ and $C_{f}$ are the initial and final configurations respectively, and $e$ is an event. Intuitively, these configurations describe the forwarding behaviors of the network before/after the update, while the event describes a phenomenon, such as the receipt of a packet at a particular switch, that triggers the update itself. Semantically, an eventtriggered consistent update ensures that for each packet:

1. the packet is forwarded consistently, i.e. it must be processed entirely by a single configuration $C_{i}$ or $C_{f}$, and

2. the update does not happen too early, meaning that if every switch traversed by the packet has not heard about the event, then the packet must be processed by $C_{i}$, and

3. the update does not happen too late, meaning that if every switch traversed by the packet has heard about the event, then the packet must be processed by $C_{f}$.

The first criterion requires that updates are consistent, which is analogous to a condition proposed previously by Reitblatt et al. [33]. However, a consistent update alone would not provide the necessary guarantees for the stateful firewall example, as it applies only to a single packet, and not to multiple packets in a bidirectional flow. The last two criteria relate the packet-processing behavior on each switch to the events it has "heard about." Note that these criteria leave substantial flexibility for implementations: packets that do not satisfy the second or third condition can be processed by either the preceding or following configuration. It remains to define what it means for a switch $s$ to have "heard about" an event $e$ that occurred at switch $t$ (assuming $s \neq t$ ). We use a causal model and say that $s$ hears about $e$ when a packet, which was processed by $t$ after $e$ occurred, is received at $s$. This can be formalized using a "happens-before" relation.

Returning to the stateful firewall, it is not hard to see that the guarantees offered by event-driven consistent updates are 
sufficient to ensure correctness of the overall application. Consider an update $C_{i} \stackrel{e}{\rightarrow} C_{f}$. In $C_{i}, H_{1}$ can send packets to $H_{4}$, but not vice-versa. In $C_{f}$, additionally $H_{4}$ can send packets to $H_{1}$. The event $e$ is the arrival at $s_{4}$ of a packet from $H_{1}$ to $H_{4}$. Before $e$ occurs, can $H_{4}$ send a packet to $H_{1}$, as is possible in $C_{f}$ ? No, since none of the switches along the necessary path have heard about the event. Now, imagine that the event $e$ occurs, and $H_{4}$ wants to send a packet to $H_{1}$ afterwards. Can $s_{4}$ drop the new packet, as it would have done in the initial configuration $C_{i}$ ? No, because the only switch the packet would traverse is $s_{4}$, and $s_{4}$ has heard about the event, meaning that the only possible correct implementation should process this new packet in $C_{f}$.

Event-Driven Transition Systems. To specify event-driven network programs, we use labeled transition systems called event-driven transition systems (ETSs). In an ETS, each node is annotated with a network configuration and each edge is annotated with an event. For example, the stateful firewall application would be described as a two-state ETS, one state representing the initial configuration before $H_{1}$ has sent a packet to $H_{4}$, and another representing the configuration after this communication has occurred. There would be a transition between the states corresponding to receipt of a packet from $H_{1}$ to $H_{4}$ at $s_{4}$. This model is similar to the finite state machines used in Kinetic [21] and FAST [30]. However, whereas Kinetic uses uncoordinated updates, we impose additional constraints on our ETSs which allow them to be implemented correctly with respect to our consistency property. For example, we extend event-triggered consistent updates to sequences, requiring each sequence of transitions in the ETS to satisfy the property. For simplicity, in this paper, we focus on finite state systems and events corresponding to packet delivery. However, these are not fundamental assumptions - our design extends naturally to other notions of events, as well as infinite-state systems.

Network Event Structures. The key challenge in implementing event-driven network programs stems from the fact that at any time, the switches may have different views of the global set of events that have occurred. Hence, for a given ETS, several different updates may be enabled at a particular moment of time, and we need a way to resolve conflicts. We turn to the well-studied model of event structures [38], which allows us to constrain transitions in two ways: (1) causal dependency, which requires that an event $e_{1}$ happens before another event $e_{2}$ may occur, and (2) compatibility, which forbids sets of events that are in some sense incompatible with each other from occurring in the same execution. We present an extension called network event structure (NES), and show how an ETS can be encoded as an NES.

Locality. While event-driven consistent updates require immediate responses to local events (as in the firewall), they do not require immediate reactions to events "at a distance." This is achieved by two aspects of our definitions.
The first defining aspect of our locality requirements involves the happens-before ("heard-about") relation in eventdriven consistent update. For example, the receipt of a packet in New York can not immediately affect the behavior of switches in London. Intuitively, this makes sense: requiring "immediate" reaction to remote events would force synchronization between switches and buffering of packets, leading to unacceptable performance penalties. Event-driven consistent update only requires the switches in London to react after they have heard about the event in New York.

The second defining aspect of our locality requirements involves the compatibility constraints in NESs. Suppose that New York sends packets to London and Paris, but the program requires transitioning to a different global state based on who received a packet first. Clearly, it would be impossible to implement this behavior without significant coordination. However, suppose New York and Philadelphia are sending packets to London, and the program requires transitioning to a different global state based on whose packet was received first in London. This behavior is easily implementable since the choice is local to London. We use NESs to rule out non-local incompatible events-specifically, we require that incompatible events must occur at the same switch.

Our approach gives consistency guarantees even when an event occurs at a switch different from the one that will be updated. The change will not happen "atomically" with the event that triggered it, but (a) every packet is processed by a single configuration, and (b) the configuration change occurs as dictated by event-driven consistent update (happensbefore) requirements. We show that these requirements can be implemented with minimal performance penalty.

Locality issues are an instance of the tension between consistency and availability in distributed systems, which motivates existing SDN languages to favor availability (avoiding expensive synchronization and packet buffering) over consistency (offering strong guarantees when state changes). We demonstrate that it is possible to provide the same level of availability as existing systems, while providing a natural consistency condition that is powerful enough to build many applications. We also show that weakening the locality requirement forces us to weaken availability.

Overall, we present a new abstraction based on (i) a notion of causal consistency requiring that events are propagated between nodes, (ii) per-packet consistency governing how packets are forwarded through the network, and (iii) locality requirements. We believe this is a powerful combination that is a natural fit for building many applications.

Implementing Network Programs. NESs also provide a natural formalism for guiding an implementation technique for stateful programs. Intuitively, we need switches that can record the set of events that have been seen locally, make decisions based on those events, and transmit events to other switches. Fortunately, in the networking industry there is a trend toward more programmable data planes: mutable state 
is already supported in most switch ASICs (e.g. MAC learning tables) and is also being exposed to SDN programmers in next-generation platforms such as OpenState [5] and P4 [6]. Using these features, we can implement an NES as follows.

1. Encode the sets of events contained in the NES as flat tags that can be carried by packets and tested on switches.

2. Compile the configurations contained in the NES to a collection of forwarding tables.

3. Add "guards" to each configuration's forwarding rules to explicitly test for the tag enabling the configuration.

4. Add rules to "stamp" incoming packets with tags corresponding to the current set of events.

5. Add rules to "learn" which events have happened by reading tags on incoming packets and adding the tags in the local state to outgoing packets, as required to implement the happens-before relation.

In this paper, we prove that a system implemented in this way correctly implements an NES.

Evaluation. To evaluate our design, we built a prototype of the system described in this paper. $\dagger$ We have used this to build a number of event-driven network applications: (a) a stateful firewall, which we have already described; (b) a learning switch that floods packets going to unknown hosts along a spanning tree, but uses point-to-point forwarding for packets going to known hosts; (c) an authentication system that initially blocks incoming traffic, but allows hosts to gain access to the internal network by sending packet probes to a predefined sequence of ports; (d) a bandwidth cap that disables access to an external network after seeing a certain number of packets; and (e) an intrusion detection system that allows all traffic until seeing a sequence of internal hosts being contacted in a suspicious order. We have also built a synthetic application that forwards packets around a ring topology, to evaluate update scalability. We developed these applications in an extended version of NetKAT which we call Stateful NetKAT. Our experiments show that our implementation technique provides competitive performance on several important metrics while ensuring important consistency properties. We draw several conclusions. (1) Eventdriven consistent update allow programmers to easily write real-world network applications and get the correct behavior, whereas approaches relying only on uncoordinated consistency guarantees do not. (2) The performance overhead of maintaining state and manipulating tags (measured in bandwidth) is within $6 \%$ of an implementation that uses only uncoordinated update. (3) There is an optimization that exploits common structure in rules across states to reduce the number of rules installed on switches. In our experiments, a basic heuristic version of this optimization resulted in a $32-37 \%$ reduction in the number of rules required on average.

Summary. Our main contributions are as follows.

$\dagger$ The PLDI 2016 Artifact Evaluation Committee (AEC) found that our prototype system "met or exceeded expectations."
- We propose a new semantic correctness condition for dynamic network programs called event-driven consistent update that balances the need for immediate response with the need to avoid costly synchronization and buffering of packets. Our consistency property generalizes the guarantees offered by consistent updates, and is as strong as possible without sacrificing availability.

- We propose network event structures to capture causal dependencies and compatibility between events, and show how to implement these using SDN functionality.

- We describe a compiler based on a stateful extension of NetKAT, and present optimizations that reduce the overhead of implementing such stateful programs.

- We conduct experiments showing that our approach gives well-defined consistency guarantees, while avoiding expensive synchronization and packet buffering.

The rest of this paper is structured as follows: $\S 2$ formalizes event-driven consistent updates; $\S 3$ defines event transition systems, network event structures, and Stateful NetKAT; $\$ 4$ describes our implementation; and $\S 5$ presents experiments. We discuss related/future work in $\S 6-7$, and conclude in $\S 8$.

\section{Event-Driven Network Behavior}

This section presents our new consistency model for stateful network programs: event-driven consistent update.

Preliminaries. A packet pkt is a record of fields $\left\{f_{1} ; f_{2}\right.$; $\left.\cdots ; f_{n}\right\}$, where fields $f$ represent properties such as source and destination address, protocol type, etc. The (numeric) values of fields are accessed via the notation pkt.f, and field updates are denoted $p k t[f \leftarrow n]$. A switch $s w$ is a node in the network with one or more ports pt. A host is a switch that can be a source or a sink of packets. A location $l$ is a switch-port pair $n: m$. Locations may be connected by (unidirectional) physical links $\left(l_{s r c}, l_{d s t}\right)$ in the topology.

Packet forwarding is dictated by a network configuration $C$. A located packet $l p=(p k t, s w, p t)$ is a tuple consisting of a packet and a location $s w: p t$. We model $C$ as a relation on located packets: if $C\left(l p, l p^{\prime}\right)$, then the network maps $l p$ to $l p^{\prime}$, possibly changing its location and rewriting some of its fields. Since $C$ is a relation, it allows multiple output packets to be generated from a single input. In a real network, the configuration only forwards packets between ports within each individual switch, but for convenience, we assume that our $C$ also captures link behavior (forwarding between switches $)$, i.e. $C\left(\left(p k t, n_{1}, m_{1}\right),\left(p k t, n_{2}, m_{2}\right)\right)$ holds for each link $\left(n_{1}: m_{1}, n_{2}: m_{2}\right)$. We refer to a sequence of located packets that starts at a host and can be produced by $C$ as a packet trace, using Traces $(C)$ to denote the set of all such packet traces. We let $\mathcal{C}$ be the set of all configurations.

Consider a tuple $n t r=\left(l p_{0} l p_{1} \cdots, T\right)$, where the first component is a sequence of located packets, and each $t \in T$ is an increasing sequence of indices corresponding to located packets in the sequence. We call such a tuple a network trace if and only if the following conditions hold: 
1. for each $l p_{j}$, we have $j \in t$ for some $t \in T$, and

2. for each $t=\left(k_{0} k_{1} \cdots\right) \in T, l p_{k_{0}}$ is at a host, and $\exists C \in \mathcal{C}$ such that $C\left(l p_{k_{i}}, l p_{k_{i+1}}\right)$ holds for all $i$, and

3. if we consider the graph $G$ with nodes $\{k:(\exists t \in$ $T: k \in t)\}$ and edges $\left\{\left(k_{i}, k_{i+1}\right):(\exists t \in T: t=\right.$ $\left.\left.k_{0} k_{1} \cdots k_{i} k_{i+1} \cdots\right)\right\}$, then $G$ is a family of trees rooted at $K=\left\{k_{0}:\left(\exists t \in T: t=k_{0} \cdots\right)\right\}$.

We will use $n t r \downarrow k$ to denote the set $\{t \in T: k \in t\}$, and when $t=\left(k_{0} k_{1} \cdots\right) \in T$, we can use similar notation $n t r \downarrow t$ to denote the packet trace $l p_{k_{0}} l p_{k_{1}} \cdots$. Intuitively, we have defined a network trace to be an interleaving of these packet traces (the packet traces form the family of trees because, as previously mentioned, the configuration allows multiple output packets from a single input packet). Ultimately, we will introduce a consistency definition that dictates which interleavings of packet traces are correct.

We now define how the network changes its configuration in response to events. An event e is a tuple $(\varphi, s w, p t)_{\text {eid }}$, where eid is an (optional) event identifier and $\varphi$ is a firstorder formula over fields. Events model the arrival of a packet satisfying $\varphi$ (denoted $p k t \models \varphi$ ) at location sw:pt. Note that we could have other types of events-anything that a switch can detect could be an event-but for simplicity, we focus on packet events. We say that a located packet $l p=$ $\left(p k t, s w^{\prime}, p t^{\prime}\right)$ matches an event $e=(\varphi, s w, p t)$ (denoted by $l p \models e)$ if and only if $s w=s w^{\prime} \wedge p t=p t^{\prime} \wedge p k t \models \varphi$.

Definition 1 (Happens-before relation $\prec_{n t r}$ ). Given a network trace $n t r=\left(l p_{0} l p_{1} \cdots, T\right)$, the happens-before relation $\prec_{n t r}$ is the least partial order on located packets that

- respects the total order induced by ntr at switches, i.e., $\forall i, j: l p_{i} \prec l p_{j} \Leftarrow i<j \wedge l p_{i}=(p k t, s w, p t) \wedge l p_{j}=$ $\left(p k t^{\prime}, s w, p t^{\prime}\right)$, and

- respects the total order induced by ntr for each packet, i.e., $\forall i, j: l p_{i} \prec l p_{j} \Leftarrow i<j \wedge \exists t \in T: i \in t \wedge j \in t$.

Event-Driven Consistent Update. In Section 1, we informally defined an event-driven consistent update as a triple $C_{i} \stackrel{e}{\rightarrow} C_{f}$ consisting of an initial configuration $C_{i}$, event $e$, and final configuration $C_{f}$. Here, we formalize that definition in a way that describes sequences of events and configurations (in the single-event case, this formal definition is equivalent to the informal one). We denote an event-driven consistent update as a pair $(U, \mathcal{E})$, where $U$ is a sequence $C_{0} \stackrel{e_{0}}{\longrightarrow} C_{1} \stackrel{e_{1}}{\longrightarrow} \cdots \stackrel{e_{n}}{\longrightarrow} C_{n+1}$, and $\left\{e_{0}, \cdots, e_{n}\right\} \subseteq \mathcal{E}$.

Let $n t r=\left(l p_{0} l p_{1} \cdots, T\right)$ be a network trace. Given an event-driven consistent update $(U, \mathcal{E})$, we need the indices where the events from $U$ first occurred. Specifically, we wish to find the sequence $k_{0}, \cdots, k_{n}$ where $l p_{j}$ does not match any $e \in \mathcal{E}$ for any $j>k_{n}$, and the following properties hold for all $0 \leq i \leq n$ (assuming $k_{(-1)}=-1$ for convenience):

- $k_{i}>k_{i-1}$, and

- $l p_{k_{i}}$ matches $e_{i}$, and for all $j$, if $k_{i-1}<j<k_{i}$ then $l p_{j}$ does not match $e_{i}$ (i.e., $k_{i}$ is the first occurrence of $e_{i}$ after the index $k_{i-1}$ ), and

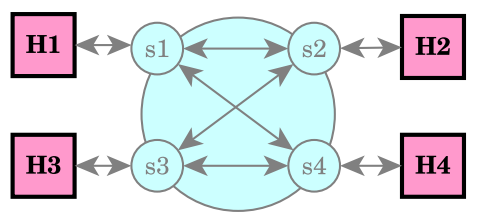

Figure 2: Example topology with four switches and hosts.

- $\exists t \in n t r \downarrow k_{i}$ such that $t$ is in $\operatorname{Traces}\left(C_{i}\right)$ (intuitively, the event $e_{i}$ can be triggered only by a packet processed in the immediately preceding configuration).

If such a sequence exists, it is unique, and we denote it by $F O(n t r, U)$, shorthand for "first occurrences."

Definition 2 (Event-driven consistent update correctness). A network trace $n t r=\left(l p_{0} l p_{1} \cdots, T\right)$ is correct with respect to an event-driven consistent update $U=C_{0} \stackrel{e_{0}}{\longrightarrow} C_{1} \stackrel{e_{1}}{\longrightarrow}$ $\ldots \stackrel{e_{n}}{\longrightarrow} C_{n+1}$, if $F O(n t r, U)=k_{0}, \cdots, k_{n}$ exists, and for all $0 \leq i \leq n$, the following holds for each packet trace $n t r \downarrow t=l p_{0}^{\prime} l p_{1}^{\prime} \cdots$ where $t \in T$ :

- $n t r \downarrow t$ is in Traces $(C)$ for some $C \in\left\{C_{0}, \cdots, C_{n+1}\right\}$ (packet is processed entirely by one configuration), and

- if $\forall j: l p_{j}^{\prime} \prec l p_{k_{i}}$, then ntr $\downarrow t$ is in Traces $(C)$ for some $C \in\left\{C_{0}, \cdots, C_{i}\right\}$ (the packet is processed entirely in a preceding configuration), and

- if $\forall j: l p_{k_{i}} \prec l p_{j}^{\prime}$, then ntr $\downarrow t$ is in Traces $(C)$ for some $C \in\left\{C_{i+1}, \cdots, C_{n+1}\right\}$ (the packet is processed entirely in a following configuration).

To illustrate, consider Figure 2. We describe an update $C_{i} \stackrel{e}{\rightarrow} C_{f}$. In the initial configuration $C_{i}$, the host $H_{1}$ can send packets to $H_{2}$, but not vice-versa. In the final configuration $C_{f}$, traffic from $H_{2}$ to $H_{1}$ is allowed. Event $e$ models the arrival to $s_{4}$ of a packet from $H_{1}$ (imagine $s_{4}$ is part of a distributed firewall). Assume that $e$ occurs, and immediately afterwards, $H_{2}$ wants to send a packet to $s_{1}$. Can $s_{2}$ drop the packet (as it would do in configuration $\left.C_{i}\right)$ ? Event-driven consistent updates allow this, as otherwise we would require $s_{2}$ to react immediately to the event at $s_{4}$, which would be an example of action at a distance. Formally, the occurrence of $e$ is not in a happens-before relation with the arrival of the new packet to $s_{2}$. On the other hand, if e.g. $s_{4}$ forwards some packets to $s_{1}$ and $s_{2}$ before the new packet from $H_{2}$ arrives, $s_{1}$ and $s_{2}$ would be required to change their configurations, and the packet would be allowed to reach $H_{1}$.

Network Event Structures. As we have seen, event-driven consistent updates specify how the network should behave during a sequence of updates triggered by events, but additionally, we want the ability to capture constraints between the events themselves. For example, we might wish to say that $e_{2}$ can only happen after $e_{1}$ has occurred, or that $e_{2}$ and $e_{3}$ cannot both occur in the same network trace.

To model such constraints, we turn to the event structures model introduced by Winskel [38]. Intuitively, an event structure endows a set of events $\mathcal{E}$ with (a) a consistency 
predicate (con) specifying which events are allowed to occur in the same sequence, and (b) an enabling relation $(\vdash)$ specifying a (partial) order in which events can occur. This is formalized in the following definition (note that we use $\subseteq_{\text {fin }}$ to mean "finite subset," and $\left.\mathcal{P}_{\text {fin }}(X)=\left\{Y: Y \subseteq_{\text {fin }} \mathcal{P}(X)\right\}\right)$.

Definition 3 (Event structure). An event structure is a tuple $(\mathcal{E}$, con,$\vdash)$ where:

- $\mathcal{E}$ is a set of events,

- con $:\left(\mathcal{P}_{\text {fin }}(\mathcal{E}) \rightarrow\right.$ Boolean $)$ is a consistency predicate that satisfies $\operatorname{con}(X) \wedge Y \subseteq X \Longrightarrow \operatorname{con}(Y)$,

- $\vdash:(\mathcal{P}(\mathcal{E}) \times \mathcal{E} \rightarrow$ Boolean $)$ is an enabling relation that satisfies $(X \vdash e) \wedge X \subseteq Y \Longrightarrow(Y \vdash e)$.

An event structure can be seen as defining a transition system whose states are subsets of $\mathcal{E}$ that are consistent and reachable via the enabling relation. We refer to such a subset an as an event-set (called "configuration" in [38]).

Definition 4 (Event-set of an event structure). Given an event structure $N=(\mathcal{E}$, con,$\vdash)$, an event-set of $N$ is any subset $X \subseteq \mathcal{E}$ which is: (a) consistent: $\forall Y \subseteq$ fin $X$, con $(Y)$ holds, and (b) reachable via the enabling relation: for each $e \in X$, there exists $e_{0}, e_{1}, \cdots, e_{n} \in X$ where $e_{n}=e$ and $\emptyset \vdash\left\{e_{0}\right\}$ and $\left\{e_{0}, \cdots, e_{i-1}\right\} \vdash e_{i}$ for all $1 \leq i \leq n$.

We want to be able to specify which network configuration should be active at each event-set of the event structure. Thus, we need the following extension of event structures.

Definition 5 (Network event structure (NES)). A network event structure is a tuple $(\mathcal{E}$, con $, \vdash, g)$ where $(\mathcal{E}$, con,$\vdash)$ is an event structure, and $g:(\mathcal{P}(\mathcal{E}) \rightarrow \mathcal{C})$ maps each event-set of the event structure to a network configuration.

Correct Network Traces. We now define what it means for a network trace $n t r$ to be correct with respect to an NES $N=(\mathcal{E}$, con $, \vdash, g)$. We begin by constructing a sequence $S$ of events that is allowed by $N$. A sequence $S=e_{0} e_{1} \cdots e_{n}$ is allowed by $N$, if $\emptyset \vdash\left\{e_{0}\right\} \wedge \operatorname{con}\left(\left\{e_{0}\right\}\right)$, and $\forall 1 \leq i \leq n$ : $\left(\left\{e_{0}, e_{1}, \cdots, e_{i-1}\right\} \vdash e_{i} \wedge \operatorname{con}\left(\left\{e_{0}, e_{1}, \cdots, e_{i}\right\}\right)\right)$.

Intuitively, we say that $n t r$ is correct if there is a sequence of events allowed by $N$ which would cause $n t r$ to satisfy the event-driven consistent update condition.

Definition 6 (Correct network trace). Let $\mathcal{S}$ be the set of all sequences allowed by $N$. Formally, a network trace $n t r=$ $\left(l p_{0} l p_{1} \cdots, T\right)$ is correct with respect to $N$ if

- no $l p_{j}$ matches any $e \in \mathcal{E}$, and for all packet traces $n t r \downarrow t$ where $t \in T$, we have ntr $\downarrow t$ is in Traces $(g(\emptyset))$, or

- there exists some $e_{0} e_{1} \cdots e_{n} \in \mathcal{S}$ such that ntr is correct with respect to event-driven consistent update $\left(g(\emptyset) \stackrel{e_{0}}{\longrightarrow}\right.$ $\left.g\left(\left\{e_{0}\right\}\right) \stackrel{e_{1}}{\longrightarrow} \cdots \stackrel{e_{n}}{\longrightarrow} g\left(\left\{e_{0}, \cdots, e_{n}\right\}\right), \mathcal{E}\right)$.

Locality Restrictions for Incompatible Events. We now show how NESs can be used to impose reasonable locality restrictions. A set of events $E$ is called inconsistent if and only if $\operatorname{con}(E)$ does not hold. We use the term minimally-inconsistent to describe inconsistent sets where all proper subsets are not inconsistent. An NES $N$ is called locally-determined if and only if for each of its minimallyinconsistent sets $E$, all events in $E$ happen at the same switch (i.e., $\exists s w \forall e_{i} \in E: e_{i}=\left(\varphi_{i}, s w, p t_{i}\right)$ ). To illustrate the need for the locally-determined property, let us consider the following two programs, $P_{1}$ and $P_{2}$.

- Program $P_{1}$ : Recall that two events are inconsistent if either of them can happen, but both cannot happen in the same execution. Consider the topology shown in Figure 2 and suppose this program requires that $H_{2}$ and $H_{4}$ can both receive packets from $H_{1}$, but only the first one to receive a packet is allowed to respond. There will be two events $e_{1}$ and $e_{2}$, with $e_{1}$ the arrival of a packet from $H_{1}$ at $s_{2}$, and $e_{2}$ the arrival of a packet from $H_{1}$ at $s_{4}$. These events are always enabled, but the set $\left\{e_{1}, e_{2}\right\}$ is not consistent, i.e. $\operatorname{con}\left(\left\{e_{1}, e_{2}\right\}\right)$ does not hold. This models the fact that at most one of the events can take effect. These events happen at different switches-making sure that at most one of the events takes effect would necessitate information to be propagated instantaneously "at a distance." In implementations, this would require using inefficient mechanisms (synchronization and/or packet buffering). Our locality restriction is a clean condition which ensures that the NES is efficiently implementable.

- Program $P_{2}$ : Consider a different program where $H_{2}$ can send traffic to one of the two hosts $H_{1}, H_{3}$ that sends it a packet first. The two events (a packet from $H_{1}$ arriving at $s_{2}$, and a packet from $H_{3}$ arriving at $s_{2}$ ) are still inconsistent, but inconsistency does not cause problems in this case, because both events happen at the same switch (the switch can determine which one was first).

In contrast to our approach, an uncoordinated update approach improperly handles locality issues, mainly because it does not guarantee when the configuration change occurs. Consider the program $P_{1}$ again, and consider the (likely) scenario where events $e_{1}$ and $e_{2}$ happen nearly simultaneously. In an uncoordinated approach, this could result in switch $s_{2}$ hearing about $e_{1}, e_{2}$ (in that order), and $s_{4}$ hearing about $e_{2}, e_{1}$ (in that order), meaning the two switches would have conflicting ideas of which event was "first" (i.e. the switches would be in conflicting states, and this conflict cannot be resolved). In our implementation, we would require $e_{1}$ and $e_{2}$ to occur at the same switch, guaranteeing that we never see such a conflicting mix of states.

Strengthening Consistency. We now show that strengthening the consistency conditions imposed by NESs would lead to lower availability, as it would lead to the need for expensive synchronization, packet buffering, etc. First, we will try to remove the locally-determined condition, and second, we will try to obtain a strengthened consistency condition. The proof of the following theorem is an adaptation of the proof of the CAP theorem [7], as presented in [13]. The idea is that in asynchronous network communication, a switch might need to wait arbitrarily long to hear about an event. 
Lemma 1. In general, it is impossible to implement an NES that does not have the locally-determined condition while guaranteeing that switches process each packet within an a priori given time bound.

Proof Sketch. Consider a simple NES, with event sets $\emptyset$, $\left\{e_{1}\right\},\left\{e_{2}\right\}$, and where $\left\{e_{1}\right\}$ and $\left\{e_{2}\right\}$ are both enabled from $\emptyset$. Assume that $\operatorname{con}\left(\left\{e_{1}, e_{2}\right\}\right)$ does not hold, and that $e_{1}$ can happen at switch $A$ and $e_{2}$ can happen at switch $B$ (i.e., the locally-determined condition does not hold).

Because the communication is asynchronous, there is no a priori bound on how long the communication between switches can take. When a packet $p$ that matches $e_{2}$ arrives at the switch $B$, the switch must distinguish the following two cases: (\#1) event $e_{1}$ has occurred at $A$ (and thus $p$ does not cause $e_{2}$ ), or (\#2) event $e_{1}$ has not occurred at $A$ (and thus $p$ causes $e_{2}$ ). No matter how long $B$ waits, it cannot distinguish these two cases, and hence, when a packet that matches $e_{2}$ arrives to $B$, the switch $B$ cannot correctly decide whether to continue as if $e_{2}$ has happened. It has the choice to either eventually decide (and risk the wrong decision), or to buffer the packet that matches $e_{2}$.

We now ask whether we can strengthen the event-driven consistent update definition. We define strong update as an update $C_{1} \stackrel{e}{\rightarrow} C_{2}$ such that immediately after $e$ occurred, the network processes all incoming packets in $C_{2}$. We obtain the following lemma by the same reasoning as the previous one.

Lemma 2. In general, it is impossible to implement strong updates and guarantee that switches process each packet within an a priori given time bound.

Proof Sketch. Let $A$ be the switch where $e$ can happen, and let $B$ be a switch on which the configurations $C_{1}, C_{2}$ differ. For $A$ and $B$, the same argument as in the previous lemma shows that $B$ must either risk the wrong decision on whether to process packets using $C_{1}$ or $C_{2}$, or buffer packets.

\section{Programming with Events}

The correctness condition we described in the previous section offers useful application-level guarantees to network programmers. At a high level, the programmer is freed from thinking about interleavings of packets/events and responses to events (configuration updates). She can think in terms of our consistency model-each packet is processed in a single configuration, and packets entering "after" an event will be processed in the new configuration (similar to causal consistency). An important consequence is that the response to an event is immediate with respect to a given flow if the event is handled at that flow's ingress switch.

With this consistency model in mind, programmers can proceed by specifying the desired event-driven program behavior using network event structures. This section introduces an intuitive method for building NESs using simple transition systems where nodes correspond to configurations

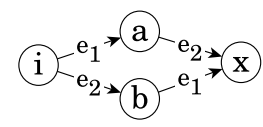

(a)

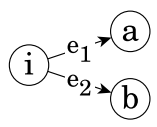

(b)

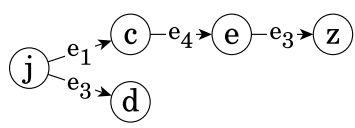

(c)
Figure 3: Event-driven transition systems.

and edges correspond to events. We also present a network programming language based on NetKAT that provides a compact notation for specifying both the transition system and the configurations at the nodes.

\subsection{Event-Driven Transition Systems}

Definition 7 (Event-driven Transition System). An eventdriven transition system (ETS) is a graph $\left(V, D, v_{0}\right)$, in which $V$ is a set of vertices, each labeled by a configuration; $D \subseteq V \times V$ is a set of edges, each labeled by an event e; and $v_{0}$ is the initial vertex.

Consider the ETSs shown in Figure 3 (a-b). In (a), the two events are intuitively compatible - they can happen in any order, so we obtain a correct execution if both happen in different parts of the network, and different switches can have a different view of the order in which they happened. In (b), the two events are intuitively incompatible-only one of them can happen in any particular execution. Therefore, even if they happen nearly simultaneously, only one of them should take an effect. To implement this, we require the locality restriction-we need to check whether the two events happen at the same switch. We thus need to distinguish between ETSs such as (a) and (b) in Figure 3, to determine where locality restrictions must be imposed in the conversion from an ETS to an NES.

From ETSS to NESs. To convert an ETS to an NES, we first form the event sets (Definition 4) and then construct the enabling relation and consistency predicate. Given an ETS $T$, consider the set $W(T)$ of sequences of events in $T$ from the initial node to any vertex (including the empty sequence). For each sequence $p \in W(T)$, let $E(p)$ be the set of events collected along the sequence. The set $F(T)=\{E(p) \mid p \in$ $W(T)\}$ is our candidate collection of event sets. We now define conditions under which $F(T)$ gives rise to an NES.

1. We require that each set $E$ in $F(T)$ must correspond to exactly one network configuration. This holds if all paths in $W(T)$ corresponding to $E$ end at states labeled with the same configuration.

2. We require that $F(T)$ is finite-complete, i.e. for any sets $E_{1}, E_{2}, \cdots, E_{n}$ where each $E_{i} \in F(T)$, if there is a set $E^{\prime} \in F(T)$ which contains every $E_{i}$ (an upper bound for the sets $E_{i}$ ), then the set $E_{l u b}=\cup_{i} E_{i}$ (the least upper bound for the $E_{i}$ ) must also be in $F(T)$. For example, consider the ETS in Figure 3(c), which violates this condition since the event-sets $E_{1}=\left\{e_{1}\right\}$ and $E_{2}=\left\{e_{3}\right\}$ are both subsets of $\left\{e_{1}, e_{4}, e_{3}\right\}$, but there is no event-set of the form $E_{1} \cup E_{2}=\left\{e_{1}, e_{3}\right\}$. 
In [38], such a collection $F(T)$ is called a family of configurations. Our condition (2) is condition (i) in Theorem 1.1.9 in [38] (conditions (ii)-(iii) are satisfied by construction).

Given an ETS $T$, it is not difficult to confirm the above conditions statically. They can be checked using straightforward graph algorithms, and any problematic vertices or edges in $T$ can be indicated to the programmer. The development of efficient checking algorithms is left for future work.

We build the con and $\vdash$ relations of an NES from the family $F(T)$, using Theorem 1.1.12. of [38]. Specifically, predicate con can be defined by declaring all sets in $F(T)$ as consistent, and for $\vdash$, we take the smallest relation satisfying the constraints $\emptyset \vdash e \Longleftrightarrow\{e\} \in F(T)$ and $X^{\prime} \vdash e \Longleftrightarrow$ $\left(X^{\prime} \in\right.$ con $) \wedge\left(\left(X^{\prime} \cup\{e\}\right) \in F(T) \vee\left(\exists X \subseteq X^{\prime}: X \vdash e\right)\right)$.

After obtaining an NES, deciding whether it satisfies the locality restriction is easy: we check whether the NES is locally determined (see Section 2), verifying for each minimally-inconsistent set that the locality restriction holds. Again, we leave the efficiency of this check for future work.

Loops in ETSs. If there are loops in the ETS T, the previous definition needs to be slightly modified, because we need to "rename" events encountered multiple times in the same execution. This gives rise to an NES where each eventset is finite, but the NES itself might be infinite (and thus can only be computed lazily). If we have the ability to store and communicate unbounded (but finite) event-sets in the network runtime, then no modifications are needed to handle infinite NESs in the implementation (which is described in Section 4). Otherwise, there are various correct overapproximations we could use, such as computing the stronglyconnected components (SCCs) of the ETS, enforcing the locality restriction on events in each (non-singleton) SCC, and requiring the implementation to attach timestamps on occurrences of events in those SCCs. For simplicity of the presentation, we will consider only loop-free ETSs in this paper.

\subsection{Stateful NetKAT}

NetKAT [2] is a domain-specific language for specifying network behavior. It has semantics based on Kleene Algebra with Tests (KAT), and a sound and complete equational theory that enables formal reasoning about programs. Operationally, a NetKAT program behaves as a function which takes as input a single packet, and uses tests, fieldassignments, sequencing, and union to produce a set of "histories" corresponding to the packet's traces.

Standard NetKAT does not support mutable state. Each packet is processed in isolation using the function described by the program. In other words, we can use a standard NetKAT program for specifying individual network configurations, but not event-driven configuration changes. We describe a stateful variant of NetKAT which allows us to compactly specify a collection of network configurations, as well as the event-driven relationships between them (i.e. an ETS). This variant preserves the existing equational theory of the

$$
\begin{aligned}
& f \in \text { Field } \\
& n \in \mathbb{N} \\
& x \quad::=f \mid \mathbf{p t} \\
& a, b::=\text { true } \mid \text { false }|x=n| \mathbf{s w}=n \mid \operatorname{state}(n)=n \\
& |a \vee b| a \wedge b \mid \neg a \\
& p, q::=a|x \leftarrow n| p+q|p ; q| p * \\
& |(n: n) \rightarrow(n: n)|(n: n) \rightarrow(n: n) \rightarrow\langle\text { state }(n) \leftarrow n\rangle
\end{aligned}
$$

Figure 4: Stateful NetKAT: syntax.

$$
\begin{aligned}
& \llbracket \operatorname{state}(m)=n \rrbracket_{\vec{k}} \triangleq \begin{cases}\llbracket \text { true } \rrbracket_{\vec{k}} & \text { if } \vec{k}(m)=n \\
\llbracket \text { false } \rrbracket_{\vec{k}} & \text { otherwise }\end{cases} \\
& \llbracket(a: b) \rightarrow(c: d) \rightarrow\langle\operatorname{state}(m) \leftarrow n\rangle \rrbracket_{\vec{k}} \triangleq \llbracket(a: b) \rightarrow(c: d) \rrbracket_{\vec{k}}
\end{aligned}
$$

Figure 5: Stateful NetKAT: extracting NetKAT Program (state $\vec{k}$ ).

individual static configurations (though it is not a KAT itself), but also allows packets to affect processing of future packets via assignments to (and tests of) a global state. The syntax of Stateful NetKAT is shown in Figure 4. A Stateful NetKAT program is a command, which can be:

- a test, which is a formula over packet header fields (there are special fields sw and pt which test the switch- and port-location of the packet respectively),

- a field assignment $x \leftarrow n$, which modifies the (numeric) value stored in a packet's field,

- a union of commands $p+q$, which unions together the packet-processing behavior of commands $p$ and $q$,

- a command sequence $p ; q$, which runs packet-processing program $q$ on the result of $p$,

- an iteration $p *$, which is equivalent to true $+p+(p ; p)+$ $(p ; p ; p)+\cdots$,

- or a link definition $\left(n_{1}: m_{1}\right) \rightarrow\left(n_{2}: m_{2}\right)$, which forwards a packet from port $m_{1}$ at switch $n_{1}$ across a physical link to port $m_{2}$ at switch $n_{2}$.

The functionality described above is also provided by standard NetKAT [35]. The key distinguishing feature of our Stateful NetKAT is a special global vector-valued variable called state, which allows the programmer to represent a collection of NetKAT programs. The function shown in Figure 5 gives the standard NetKAT program $\llbracket p \rrbracket_{\vec{k}}$ corresponding to each value $\vec{k}$ of the state vector (for conciseness, we only show the non-trivial cases). We can use the NetKAT compiler [35] to generate forwarding tables (i.e. configurations) corresponding to these, which we denote $C\left(\llbracket p \rrbracket_{\vec{k}}\right)$.

\subsection{Converting Stateful NetKAT Programs to ETSs}

Now that we have the $\llbracket \cdot \rrbracket_{\vec{k}}$ function to extract the static configurations (NetKAT programs) corresponding to the vertices of an ETS, we define another function $l \cdot l_{\vec{k}}$, which produces the event-edges (Figure 6). This collects (using parameter $\varphi$ ) the conjunction of all tests seen up to a given 


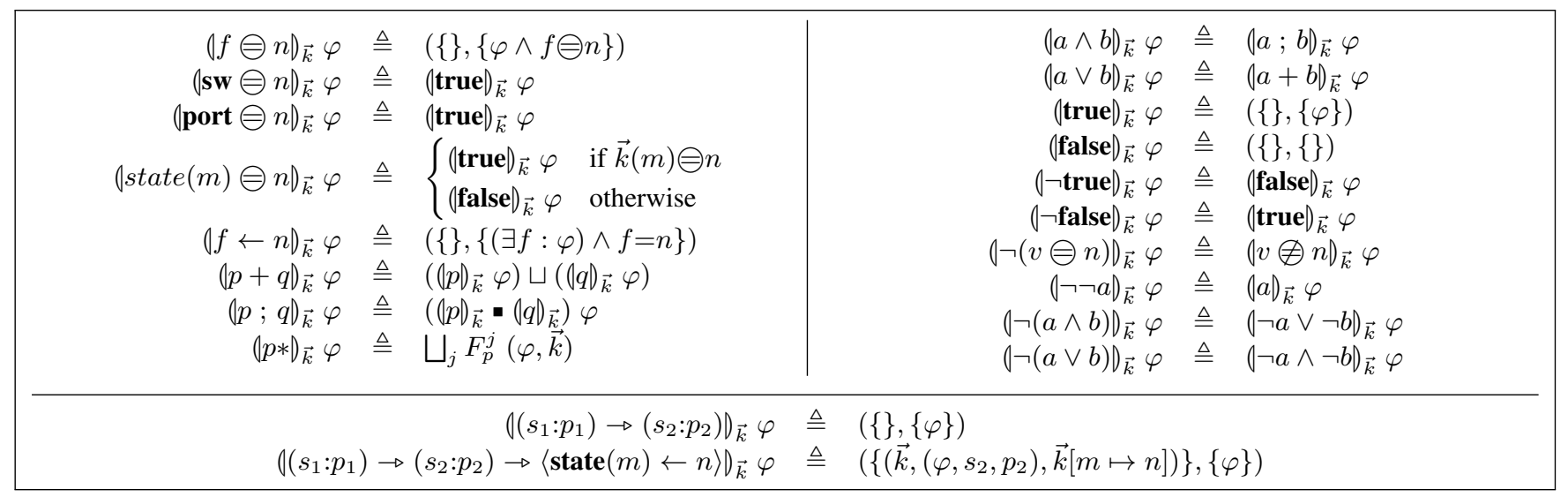

Figure 6: Stateful NetKAT: extracting event-edges from state $\vec{k}$.

program location, and records a corresponding event-edge when a state assignment command is encountered. The function returns a tuple $(D, P)$, where $D$ is a set of event-edges, and $P$ is a set of updated conjunctions of tests. In the figure, the $\sqcup$ operator denotes pointwise union of tuples, i.e. $\left(A_{1}, B_{1}, \cdots\right) \sqcup\left(A_{2}, B_{2}, \cdots\right)=\left(A_{1} \cup A_{2}, B_{1} \cup B_{2}, \cdots\right)$. The - operator denotes (pointwise) Kleisli composition, i.e. $(f \cdot g) \triangleq \bigsqcup\{g y: y \in f x\}$, and function $F$ is as follows.

$$
\begin{aligned}
F_{p}^{0}(\varphi, \vec{k}) & \triangleq(\{\},\{\varphi\}) \\
F_{p}^{j+1}(\varphi, \vec{k}) & \triangleq\left((p)_{\vec{k}} \cdot F_{p}^{j}\right) \varphi
\end{aligned}
$$

The symbol variable $\ominus$ is either equality "=" or inequality " $\neq$ ", and $\bigoplus$ is the opposite symbol with respect to $\ominus$. Given any conjunction $\varphi$ and a header field $f$, the formula $(\exists f: \varphi)$ strips all predicates of the form $(f \ominus n)$ from $\varphi$.

Using $f s t$ to denote obtaining the first element of a tuple, we can now produce the event-driven transition system for a Stateful NetKAT program $p$ with the initial state $\vec{k}_{0}$ :

$$
\begin{gathered}
\operatorname{ETS}(p) \triangleq\left(V, D, v_{0}\right) \\
\text { where } V \triangleq \bigcup_{\vec{k}}\left\{\left(\vec{k}, C\left(\llbracket p \rrbracket_{\vec{k}}\right)\right)\right\} \\
\text { and } D \triangleq f s t\left(\bigsqcup_{\vec{k}}(p p)_{\vec{k}} \text { true }\right) \\
\text { and } v_{0} \triangleq\left(\vec{k}_{0}, C\left(\llbracket p \rrbracket_{\vec{k}_{0}}\right)\right)
\end{gathered}
$$

\section{Implementing Event-Driven Programs}

Next, we show one method of implementing NESs in a real SDN, and we prove that this approach is correct-i.e., all traces followed by actual packets in the network are correct with respect to Definition 6 in Section 2. At a high level, the basic idea of our implementation strategy can be understood as follows. We assume that the switches in the network provide mutable state that can be read and written as packets are processed. Given an NES, we assign a tag to each eventset and compile to a collection of configurations whose rules are "guarded" by the appropriate tags. We then add logic that (i) updates the mutable state to record local events, (ii) stamps incoming packets with the tag for the current event- set upon ingress, and (iii) reads the tags carried by packets, and updates the event-set at subsequent switches.

\subsection{Implementation Building Blocks}

Static Configurations. The NES contains a set of network configurations that need to be installed as flow tables on switches. In addition, we must be able to transition to a new configuration in response to a local event. We do this proactively, installing all of the needed rules on switches in advance, with each rule guarded by its configuration's ID. This has a disadvantage of being less efficient in terms of rulespace usage, but an advantage of allowing quick configuration changes. In Section 5.3, we discuss an approach for addressing the space-usage issue by sharing rules between configurations. Our implementation strategy encodes each event-set in the NES as an integer, so a single unused packet header field (or single register on switches) can be used. This keeps the overhead low, even for very large programs.

Stateful Switches. Emerging data-plane languages such as P4 [6] and OpenState [5] are beginning to feature advanced functionality such as customizable parsing, stateful memories, etc. We assume that our switches support (1) modifying a local register (e.g. an integer on a switch) appropriately upon receipt of a packet, and (2) making packet forwarding decisions based on the value of a register. This allows each switch to maintain a local view of the global state. Specifically, the register records the set of events the device knows have occurred. At any time, the device can receive a packet (from the controller or another device) informing it of new event occurrences, which are "unioned" into the local register (by performing a table lookup based on integer values). Currently, P4 data planes support this type of functionality.

We also assume that the switch atomically processes each packet in the order in which it was received. Such "atomic" switch operations are proposed by the "Packet Transactions" $\mathrm{P} 4$ extension [34]. Because the P4 switch platform is attracting considerable attention (even spawning its own highly- 


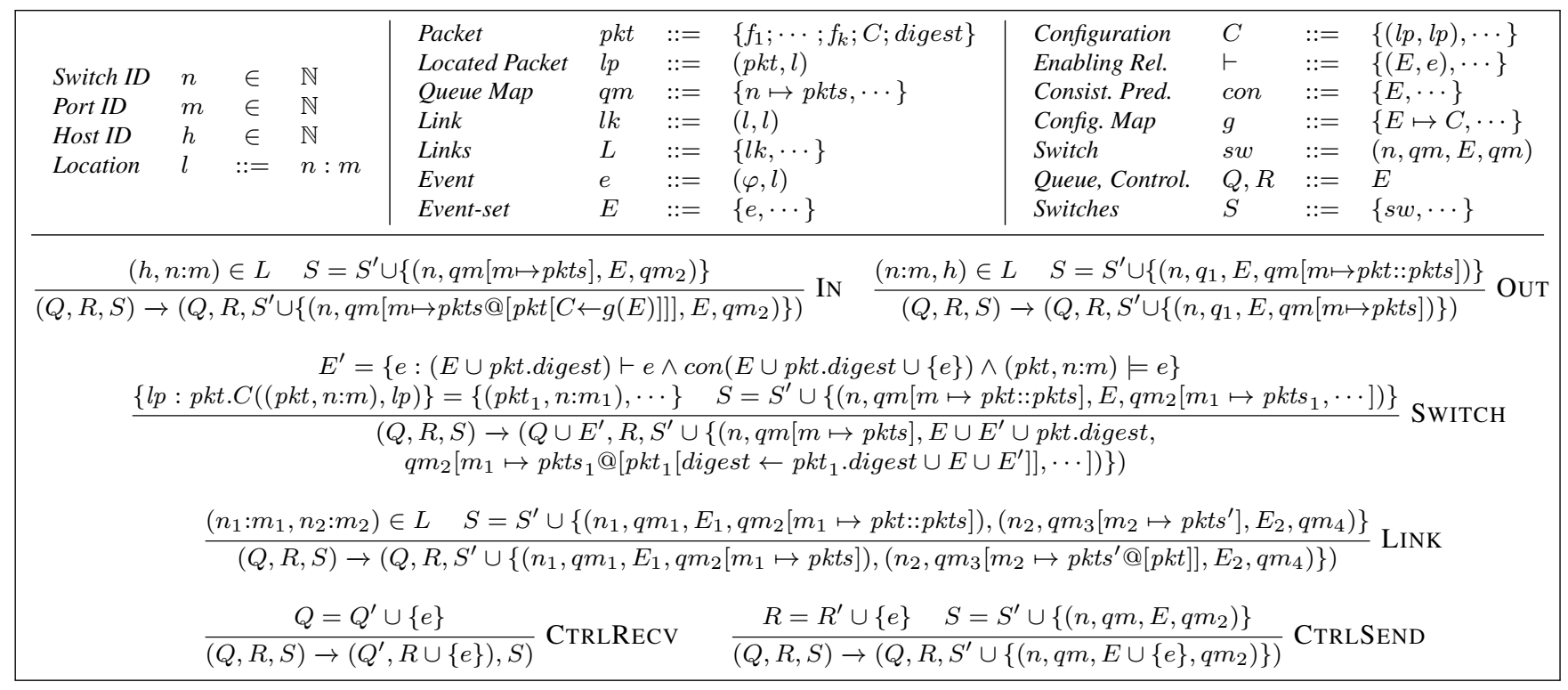

Figure 7: Implemented program semantics.

attended workshop), we feel that our assumptions are realistic for the current state-of-the-art in regards to switches.

Packet Processing. Each packet entering the network is admitted from a host to a port on an edge switch. The configuration ID $j$ corresponding to the device's view of the global state is assigned to the packet's version number field. The packet will processed only by $j$-guarded rules throughout its lifetime. Packets also carry a digest encoding the set of events the packet has heard about so far (i.e. the packet's view of the global state). If the packet passes through a device which has heard about additional events, the packet's digest is updated accordingly. Similarly, if the packet's digest contains events not yet heard about by the device, the latter adds them to its view of the state. When a packet triggers an event, that event is immediately added to the packet's digest, as well as to the state of the device where the event was detected. The controller is then notified about the event. Optionally (as an optimization), the controller can periodically broadcast its view of the global state to all switches, in order to speed up dissemination of the state.

\subsection{Operational Model}

We formalize the above via operational semantics for the global behavior of the network as it executes an NES. Each state in Figure 7 has the form $(Q, R, S)$, with a controller queue $Q$, a controller $R$, and set of switches $S$. Both the controller queue and controller are a set of events, and initially, $R=Q=\emptyset$. Each switch $s \in S$ is a tuple $\left(n, q m_{\text {in }}, E, q m_{\text {out }}\right)$, where $n$ is the switch ID, $q m_{\text {in }}, q m_{\text {out }}$ are the input/output queue maps (mapping port IDs to packet queues). Map updates are denoted $q m[m \mapsto p k t s]$. The event-set $E$ represents a switch's view of what events have occurred. A packet's digest is denoted pkt.digest, and the configuration corresponding to its version number is denoted $p k t . C$. The rules in Figure 7 can be summarized as follows.

- IN/OUT: move a packet between a host and edge port.

- SWITCH: process a packet by first adding new events from the packet's digest to the local state, then checking if the packet's arrival matches an event $e$ enabled by the NES and updating the state and packet digest if so, and finally updating the digest with other local events.

- LINK: move a packet across a physical link.

- CTRLRECV: bring an event from the controller queue into the controller.

- CTRLSEND: update the local state of the switches.

\subsection{Correctness of the Implementation}

We now prove the correctness of our implementation. Formally, we show that the operational semantics generates correct traces, as defined in Section 2.

Lemma 3 (Global Consistency). Given a locally-determined network event structure $N$, for an execution of the implementation $\left(Q_{1}, R_{1}, S_{1}\right)\left(Q_{2}, R_{2}, S_{2}\right) \cdots\left(Q_{m}, R_{m}, S_{m}\right)$, the event-set $Q_{i} \cup R_{i}$ is consistent for all $1 \leq i \leq m$.

Proof Sketch. We first show that if an inconsistent set $Y$ where $|Y|>1$ satisfies the locality restriction (i.e. all of its events are handled at the same switch), then $Y \subseteq R_{i} \cup Q_{i}$ is not possible for any $i$ (the SWITCH rule ensures that multiple events from $Y$ could not have been sent to the controller).

We proceed by induction over $m$, the trace length, noting that the base case $Q_{0} \cup R_{0}=\emptyset$ is consistent. Assume that the implementation adds an $e$ (via SWITCH) to some consistent event-set $Q_{m} \cup R_{m}$, producing an inconsistent set. We look at the minimally-inconsistent set $Y \subseteq\left(Q_{m} \cup R_{m} \cup\{e\}\right)$, and notice that the locality restriction requires all events in $Y$ to 
be detected at the same switch, so by the previous paragraph, we must have $|Y| \leq 1$. This generates a contradiction, since it would mean that either $Y=\left\{e_{0}\right\}$ or $Y \subseteq Q_{m} \cup R_{m}$, either of which would make $Y$ consistent.

Traces of the Implementation. Note that we can readily produce the network trace (Section 2) that corresponds to an implementation trace, since a single packet $p k t$ is processed at each step of Figure 7. We now present the main result of this section-executions of the implementation correspond to correct network traces (Definition 6).

Theorem 1 (Implementation Correctness). For an NES $N$, and an execution $\left(Q_{1}, R_{1}, S_{1}\right)\left(Q_{2}, R_{2}, S_{2}\right) \cdots\left(Q_{m}, R_{m}\right.$, $\left.S_{m}\right)$ of the implementation, the corresponding network trace $n$ tr is correct with respect to $N$.

Proof Sketch. The proof is by induction over the length $m$ of the execution. In the induction step, we show that (1) the SWITCH rule can only produce consistent event-sets (this follows directly from Lemma 3), and (2) when the IN rule tags a packet $p k t$ based on the local event-set $E$, that $E$ consists of exactly the events that happened before $p k t$ arrived (as ordered by the happens-before relation).

\section{Implementation and Evaluation}

We built a full-featured prototype implementation in OCaml.

- We implemented the compiler described in Section 3. This tool accepts a Stateful NetKAT program, and produces the corresponding NES, with a standard NetKAT program representing the configuration at each node. We interface with Frenetic's NetKAT compiler to produce flow-table rules for each of these NetKAT programs.

- We modified the OpenFlow 1.0 reference implementation to support the custom switch/controller needed to realize the runtime described in Section 4.

- We built tools to automatically generate custom Mininet scripts to bring up the programmer-specified network topology, using switches/controller running the compiled NES. We can then realistically simulate the whole system using real network traffic.

Research Questions. To evaluate our approach, we wanted to obtain answers to the following questions.

1. How useful is our approach? Does it allow programmers to easily write real-world network programs, and get the behavior they want?

2. What is the performance of our tools (compiler, etc.)?

3. How much does our correctness guarantee help? For instance, how do the running network programs compare with uncoordinated event-driven strategies?

4. How efficient are the implementations generated by our approach? For instance, what about message overhead? State-change convergence time? Number of rules used?

We address \#1-3 through case studies on real-world programming examples, and \#4 through quantitative perfor- mance measurements on simple automatically-generated programs. For the experiments, we assume that the programmer has first confirmed that the program satisfies the conditions allowing proper compilation to an NES, and we assume that the ETS has no loops. Our tool could be modified to perform these checks via basic algorithms operating on the ETS, but they have not yet been implemented in the current prototype (as mentioned in Section 3.1, developing efficient algorithms for these checks is left for future work). Our experimental platform was an Ubuntu machine with 20GB RAM and a quad-core Intel i5-4570 CPU (3.2 GHz).

To choose a representative set of realistic examples, we first studied the examples addressed in other recent stateful network programming approaches, such as SNAP [3], FlowLog [31], Kinetic [21], NetEgg [39], and FAST [30], and categorized them into three main groups:

- Protocols/Security: accessing streaming media across subnets, ARP proxy, firewall with authentication, FTP monitoring, MAC learning, stateful firewall, TCP reassembly, Virtual Machine (VM) provisioning.

- Measurement/Performance: heavy hitter detection, bandwidth cap management (uCap), connection affinity in load balancing, counting domains sharing the same IP address, counting IP addresses under the same domain, elephant flows detection, link failure recovery, load balancing, network information base (NIB), QoS in multimedia streaming, rate limiting, sampling based on flow size, Snort flowbits, super spreader detection, tracking flow-size distributions.

- Monitoring/Filtering: application detection, DNS amplification mitigation, DNS TTL change tracking, DNS tunnel detection, intrusion detection system (IDS), optimistic ACK attack detection, phishing/spam detection, selective packet dropping, sidejack attack detection, stolen laptop detection, SYN flood detection, UDP flood mitigation, walled garden.

As we will see in the following section, our current prototype system is best suited for writing programs such as the ones in the Protocols/Security category, since some of the Measurement/Performance programs require timers and/or integer counters, and some of the Monitoring/Filtering programs require complex pattern matching of (and table lookups based on) sequences of packets - functionality which we do not (yet) natively support, Thus, we have selected three examples from the first category, and one from each of the latter two, corresponding to the boldface applications in the list. We believe that these applications are representative of the basic types of behaviors seen in the other listed applications.

\subsection{Case Studies}

In the first set of experiments, we compare correct behavior (produced by our implementation strategy) with that of an uncoordinated update strategy. We simulate an uncoordinated strategy in the following way: events are sent to the controller, which pushes updates to the switches (in an un- 


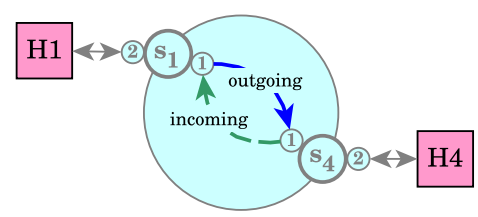

$(\mathrm{a}, \mathrm{d})$

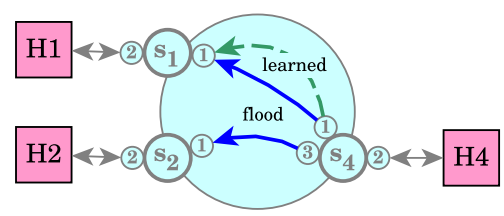

(b)

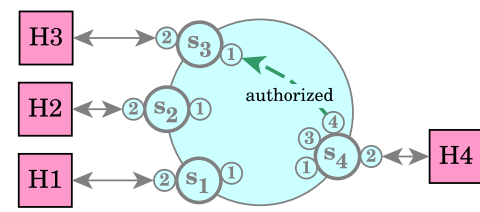

$(\mathrm{c}, \mathrm{e})$

Figure 8: Topologies: (a) Firewall, (b) Learning Switch, (c) Authentication, (d) Bandwidth Cap, (e) Intrusion Detection System.

(a)

pt $=2 \wedge$ ip_dst $=H 4 ;$ pt $\leftarrow 1 ; \quad$ (state $=[0] ;$

$(1: 1) \rightarrow(4: 1) \rightarrow\langle$ state $\leftarrow[1]\rangle+$ state $\neq[0]$

$(1: 1) \rightarrow(4: 1)) ; \mathrm{pt} \leftarrow 2$

$+\mathrm{pt}=2 \wedge$ ip_dst $=\mathrm{H} 1 ;$ state $=[1] ; \mathrm{pt} \leftarrow 1$; $(4: 1) \rightarrow(1: 1) ;$ pt $\leftarrow 2$

(b)

pt $=2 \wedge$ ip dst $=H 1 ; \quad($ pt $\leftarrow 1 ; \quad(4: 1) \rightarrow(1: 1)+$ state $=[0] ;$ pt $\leftarrow 3 ; \quad(4: 3) \rightarrow(2: 1)) ;$ pt $\leftarrow 2$

+ pt $=2 \wedge$ ip_dst $=H 4 ;$ pt $\leftarrow 1 ; \quad(1: 1) \rightarrow(4: 1) \rightarrow\langle$ state $\leftarrow[1]\rangle ; \mathrm{pt} \leftarrow 2$

$+\mathrm{pt}=2 ; \mathrm{pt} \leftarrow 1 ;(2: 1) \rightarrow(4: 3) ; \mathrm{pt} \leftarrow 2$

(c)

state $=[0] \wedge$ pt $=2 \wedge$ ip_dst $=H 1 ;$ pt $\leftarrow 1$

$(4: 1) \rightarrow(1: 1) \rightarrow\langle$ state $\leftarrow[1]\rangle ;$ pt $\leftarrow 2$

+ state $=[1] \wedge \mathrm{pt}=2 \wedge$ ip_dst $=\mathrm{H} 2 ; \mathrm{pt} \leftarrow 3$; $(4: 3) \rightarrow(2: 1) \rightarrow\langle$ state $\leftarrow[2]\rangle ;$ pt $\leftarrow 2$

+ state $=[2] \wedge$ pt $=2 \wedge$ ip_dst $=H 3 ;$ pt $\leftarrow 4 ;$ $(4: 4) \rightarrow(3: 1) ;$ pt $\leftarrow 2$

+ pt $=2 ;$ pt $\leftarrow 1 ; \quad((1: 1) \rightarrow(4: 1)+(2: 1) \rightarrow(4: 3)+$ $(3: 1) \rightarrow(4: 4)) ; \mathrm{pt} \leftarrow 2$

pt $=2 \wedge$ ip_dst $=\mathrm{H}_{4}$; pt $\leftarrow 1 ;$

state $=[0] ; \quad(1: 1) \rightarrow(4: 1) \rightarrow\langle$ state $\leftarrow[1]\rangle$

+ state $=[1] ;(1: 1) \rightarrow(4: 1) \rightarrow\langle$ state $\leftarrow[2]\rangle$

(d)

+ state $=[2] ;(1: 1) \rightarrow(4: 1) \rightarrow\langle$ state $\leftarrow[3]\rangle$

(e)

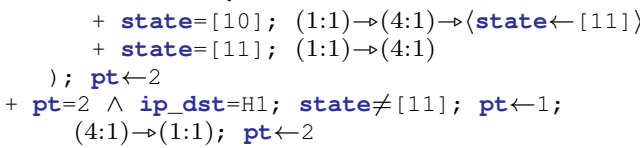

pt $=2 \wedge$ ip_dst $=H 1 ;$ pt $\leftarrow 1 ; \quad$ (state $=[0] ;$

$(4: 1) \rightarrow(1: 1) \rightarrow\langle$ state $\leftarrow[1]\rangle+$ state $\neq[0]$; $(4: 1) \rightarrow(1: 1)) ;$ pt $\leftarrow 2$

$+\mathrm{pt}=2 \wedge$ ip_dst $=\mathrm{H} 2 ;$ pt $\leftarrow 3 ; \quad($ state $=[1] ;$

$(4: 3) \rightarrow(2: 1) \rightarrow\langle$ state $\leftarrow[2]\rangle+$ state $\neq[1] ;$

$(4: 3) \rightarrow(2: 1)) ;$ pt $\leftarrow 2$

$+\mathrm{pt}=2 \wedge$ ip_dst $=\mathrm{H} 3 ;$ pt $\leftarrow 4 ;$ state $\neq[2] ;$ $(4: 4) \rightarrow(3: 1) ;$ pt $\leftarrow 2$

+ pt $=2$; pt $\leftarrow 1 ; \quad((1: 1) \rightarrow(4: 1)+(2: 1) \rightarrow(4: 3)+$ $(3: 1) \rightarrow(4: 4)) ;$ pt $\leftarrow 2$

Figure 9: Programs: (a) Firewall, (b) Learning Switch, (c) Authentication, (d) Bandwidth Cap, (e) Intrusion Detection System.

predictable order) after a few-seconds time delay. We believe this delay is reasonable because heavily using the controller and frequently updating switches can lead to delays between operations of several seconds in practice (e.g. [17] reports up to 10 s for a single switch update).

To show that problems still arise for smaller delays, in the firewall experiment described next, we varied the time delay in the uncoordinated strategy between $0 \mathrm{~ms}$ and $5000 \mathrm{~ms}$ (in increments of $100 \mathrm{~ms}$ ), running the experiment 10 times for each. We then plotted the total number of incorrectlydropped packets with respect to delay. The results are shown in Figure 10. Note that even with a very small delay, the uncoordinated strategy still always drops at least one packet.

Stateful Firewall. The example in Figures 8-9(a) is a simplified stateful firewall. It always allows "outgoing" traffic (from $\mathrm{H} 1$ to $\mathrm{H} 4$ ), but only allows "incoming" traffic (from H4 to H1) after the outside network has been contacted, i.e. "outgoing" traffic has been forwarded to $\mathrm{H} 4$.

Program $p$ corresponds to configurations $C_{[0]}=\llbracket p \rrbracket_{[0]}$ and $C_{[1]}=\llbracket p \rrbracket_{[1]}$. In the former, only outgoing traffic is allowed, and in the latter, both outgoing and incoming are allowed. The ETS has the form $\{\langle[0]\rangle \stackrel{(d s t=H 4,4: 1)}{\longrightarrow}\langle[1]\rangle\}$. The NES has the form $\left\{E_{0}=\emptyset \rightarrow E_{1}=\{(d s t=H 4,4: 1)\}\right\}$, where the $g$ is given by $g\left(E_{0}\right)=C_{[0]}, g\left(E_{1}\right)=C_{[1]}$.

The Stateful Firewall example took 0.013 s to compile, and produced a total of 18 flow-table rules. In Figure 11(a), we show that the running firewall has the expected behavior. We first try to ping H1 from H4 (the "H4-H1"/red points), which fails. Then we ping H4 from $\mathrm{H} 1$ (the "H1-H4"/orange points), which succeeds. Again we try H4-H1, and now this succeeds, since the event-triggered state change occurred.

For the uncoordinated strategy, Figure 11(b) shows that some of the H1-H4 pings get dropped (i.e. H1 does not hear back from H4), meaning the state change did not behave as if it was caused immediately upon arrival of a packet at S4.

Learning Switch. The example in Figures 8-9(b) is a simple learning switch. Traffic from $\mathrm{H} 4$ to $\mathrm{H} 1$ is flooded (sent to both $\mathrm{H} 1$ and $\mathrm{H} 2$ ), until $\mathrm{H} 4$ receives a packet from $\mathrm{H} 1$, at

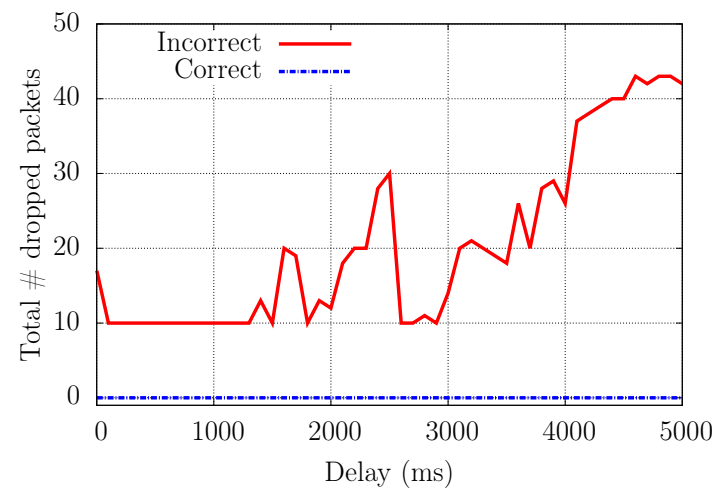

Figure 10: Stateful Firewall: impact of delay. 

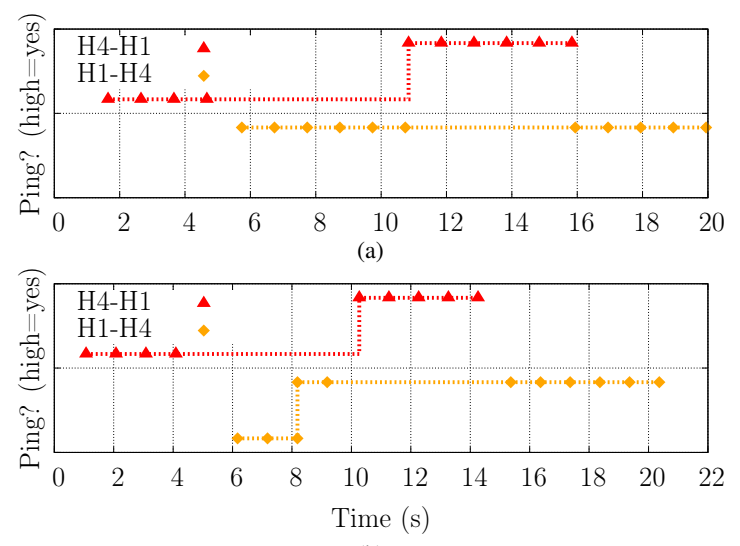

(b)

Figure 11: Stateful Firewall: (a) correct vs. (b) incorrect.

which point it "learns" the address of $\mathrm{H} 1$, and future traffic from $\mathrm{H} 4$ to $\mathrm{H} 1$ is sent only to $\mathrm{H} 1$.

This program $p$ corresponds to two configurations $C_{[0]}=$ $\llbracket p \rrbracket_{[0]}$ and $C_{[1]}=\llbracket p \rrbracket_{[1]}$. In the former, flooding occurs from $\mathrm{H} 4$, and in the latter, packets from $\mathrm{H} 4$ are forwarded directly to H1. The ETS has the form $\{\langle[0]\rangle \stackrel{(d s t=H 4,4: 1)}{\longrightarrow}\langle[1]\rangle\}$. The NES has the form $\left\{E_{0}=\emptyset \rightarrow E_{1}=\{(d s t=H 4,4: 1)\}\right\}$, where the $g$ is given by $g\left(E_{0}\right)=C_{[0]}, g\left(E_{1}\right)=C_{[1]}$.

This only allows learning for a single host (H1), but we could easily add learning for $\mathrm{H} 2$ by using a different index in the vector-valued state field: we could replace state in Figure 9(b) with state $(0)$, and union the program (using the NetKAT "+" operator) with another instance of Figure 9(b) which learns for $\mathrm{H} 2$ and uses state $(1)$.

The Learning Switch example took 0.015s to compile, and produced a total of 43 flow-table rules. We again compare the behavior of our correct implementation with that of an implementation which uses an uncoordinated update strategy. We first ping $\mathrm{H} 1$ from $\mathrm{H} 4$. Expected behavior is shown in Figure 12(a), where the first packet is flooded to both $\mathrm{H} 1$ and $\mathrm{H} 2$, but then $\mathrm{H} 4$ hears a reply from $\mathrm{H} 1$, causing
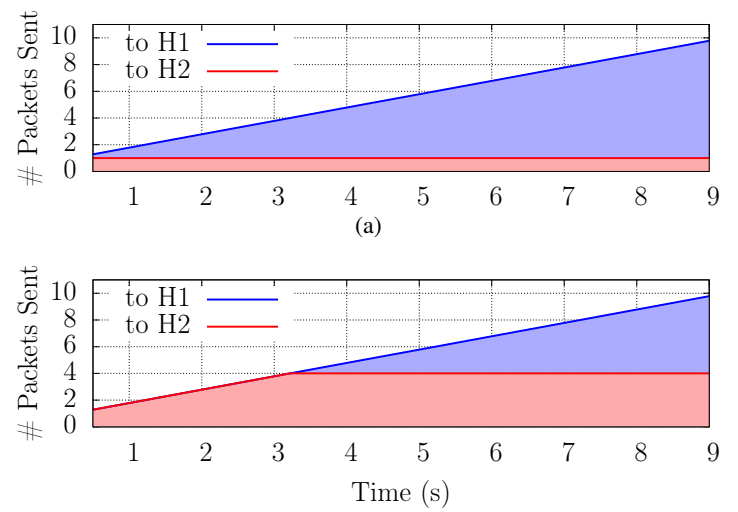

(b)

Figure 12: Learning Switch: (a) correct vs. (b) incorrect.
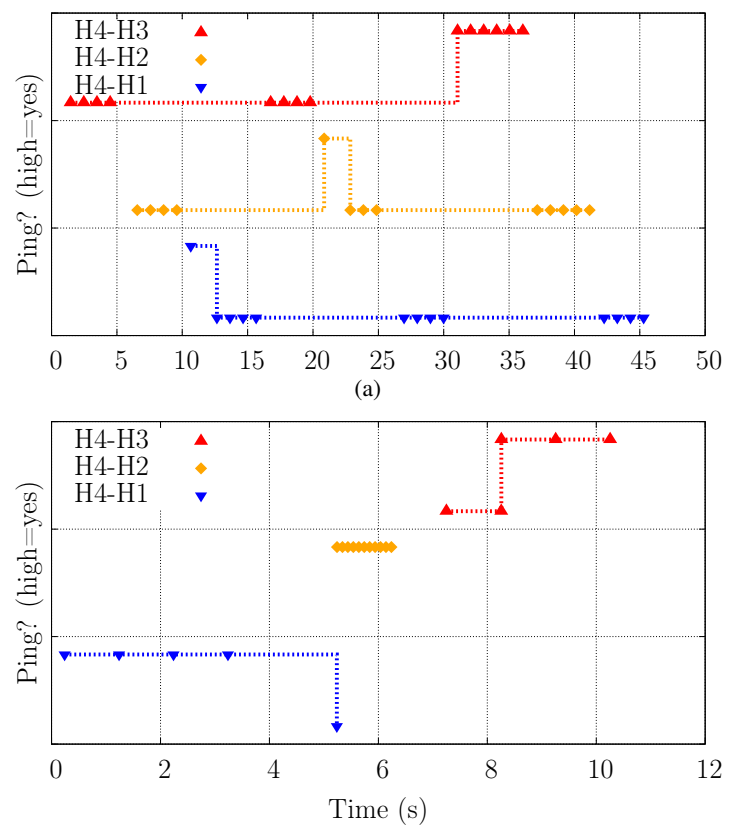

(b)

Figure 13: Authentication: (a) correct vs. (b) incorrect.

the state change (i.e. learning H1's address), and all subsequent packets are sent only to H1. In Figure 12(b), however, since the state change can be delayed, multiple packets are sent to $\mathrm{H} 2$, even after $\mathrm{H} 4$ has seen a reply from $\mathrm{H} 1$.

Authentication. In this example, shown in Figures 8-9(c), the untrusted host $\mathrm{H} 4$ wishes to contact $\mathrm{H} 3$, but can only do so after contacting $\mathrm{H} 1$ and then $\mathrm{H} 2$, in that order.

This program $p$ corresponds to three configurations: $C_{[0]}=\llbracket p \rrbracket_{[0]}$ in which only $\mathrm{H} 4-\mathrm{H} 1$ traffic is enabled, $C_{[1]}=\llbracket p \rrbracket_{[1]}$ in which only $\mathrm{H} 4-\mathrm{H} 2$ traffic is enabled, and $C_{[2]}=\llbracket p \rrbracket_{[2]}$ which finally allows $\mathrm{H} 4$ to communicate with H3. The ETS has the form $\{\langle[0]\rangle \stackrel{(d s t=H 1,1: 1)}{\longrightarrow}$ $\langle[1]\rangle \stackrel{(d s t=H 2,2: 1)}{\longrightarrow}\langle[2]\rangle\}$. The NES has the form $\left\{E_{0}=\emptyset \rightarrow\right.$ $E_{1}=\{(d s t=H 1,1: 1)\} \rightarrow E_{2}=\{(d s t=H 1,1: 1),(d s t=H 2$, $2: 1)\}\}$, where the $g$ function is given by $g\left(E_{0}\right)=C_{[0]}$, $g\left(E_{1}\right)=C_{[1]}, g\left(E_{2}\right)=C_{[2]}$.

The Authentication example took $0.017 \mathrm{~s}$ to compile, and produced a total of 72 flow-table rules. In Figure 13(a) we demonstrate the correct behavior of the program, by first trying (and failing) to ping $\mathrm{H} 3$ and $\mathrm{H} 2$ from $\mathrm{H} 4$, then successfully pinging $\mathrm{H} 1$, again failing to ping $\mathrm{H} 3$ (and $\mathrm{H} 1$ ), and finally succeeding in pinging $\mathrm{H} 3$. The incorrect (uncoordinated) implementation in Figure 13(b) allows an incorrect behavior where we can successfully ping $\mathrm{H} 1$ and then $\mathrm{H} 2$, but then fail to ping $\mathrm{H} 3$ (at least temporarily).

Bandwidth Cap. The Figure 8-9(d) example is a simplified bandwidth cap implementation. It allows "outgoing" traffic (H1-H4), but only until the limit of $n$ packets has been reached, at which point the service provider replies with a 

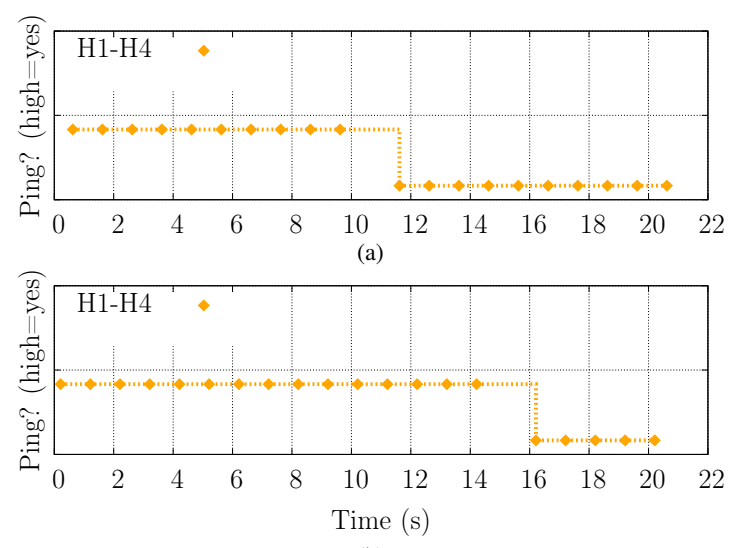

(b)

Figure 14: Bandwidth Cap: (a) correct vs. (b) incorrect.

notification message, and disallows the "incoming" path. In this experiment, we use a bandwidth cap of $n=10$ packets.

Program $p$ corresponds to configurations $C_{[0]}=\llbracket p \rrbracket_{[0]}$, $\cdots, C_{[n]}=\llbracket p \rrbracket_{[n]}$, which all allow incoming/outgoing traffic, and a configuration $C_{[n+1]}=\llbracket p \rrbracket_{[n+1]}$ which disallows the incoming traffic. The ETS has the form $\{\langle[0]\rangle \stackrel{(d s t=H 4,4: 1)}{\longrightarrow}$ $\langle[1]\rangle \stackrel{(d s t=H 4,4: 1)}{\longrightarrow} \cdots \stackrel{(d s t=H 4,4: 1)}{\longrightarrow}\langle[n+1]\rangle\}$. The NES has the form $\left\{E_{0}=\emptyset \rightarrow E_{1}=\{(d s t=H 4,4: 1)\} \rightarrow \cdots \rightarrow\right.$ $\left.E_{n+1}=\left\{(d s t=H 4,4: 1)_{0}, \cdots,(d s t=H 4,4: 1)_{n}\right\}\right\}$, where the $g$ is given by $g\left(E_{0}\right)=C_{[0]}, \cdots, g\left(E_{n+1}\right)=C_{[n+1]}$. Note that the subscripts on events in the NES event-sets (e.g. the ones in $E_{n+1}$ ) indicate "renamed" copies of the same event (as described in Section 3.1).

The Bandwidth Cap example took 0.023s to compile, and produced a total of 158 flow-table rules. In Figure 14(a), we show that the running example has the expected behavior. We send pings from $\mathrm{H} 1$ to $\mathrm{H} 4$, of which exactly 10 succeed, meaning we have reached the bandwidth cap. Using the uncoordinated update strategy in Figure 14(b), we again send pings from $\mathrm{H} 1$ to $\mathrm{H} 4$, but in this case, 15 are successful, exceeding the bandwidth cap.

Intrusion Detection System. In this example, shown in Figures 8-9(e), the external host $\mathrm{H} 4$ is initially free to communicate with the internal hosts $\mathrm{H} 1, \mathrm{H} 2$, and $\mathrm{H} 3$. However, if $\mathrm{H} 4$ begins engaging in some type of suspicious activity (in this case, beginning to scan through the hosts, e.g. contacting $\mathrm{H} 1$ and then $\mathrm{H} 2$, in that order), the activity is thwarted (in this case, by cutting off access to H3).

This program $p$ corresponds to three configurations: $C_{[0]}=\llbracket p \rrbracket_{[0]}$ and $C_{[1]}=\llbracket p \rrbracket_{[1]}$, in which all traffic is enabled, and $C_{[2]}=\llbracket p \rrbracket_{[2]}$ in which $\mathrm{H} 4-\mathrm{H} 3$ communication is disabled. The ETS has the form $\{\langle[0]\rangle \stackrel{(d s t=H 1,1: 1)}{\longrightarrow}$ $\langle[1]\rangle \stackrel{(d s t=H 2,2: 1)}{\longrightarrow}\langle[2]\rangle\}$. The NES has the form $\left\{E_{0}=\emptyset \rightarrow\right.$ $E_{1}=\{(d s t=H 1,1: 1)\} \rightarrow E_{2}=\{(d s t=H 1,1: 1),(d s t=H 2$, $2: 1)\}\}$, where the $g$ function is given by $g\left(E_{0}\right)=C_{[0]}$, $g\left(E_{1}\right)=C_{[1]}, g\left(E_{2}\right)=C_{[2]}$.
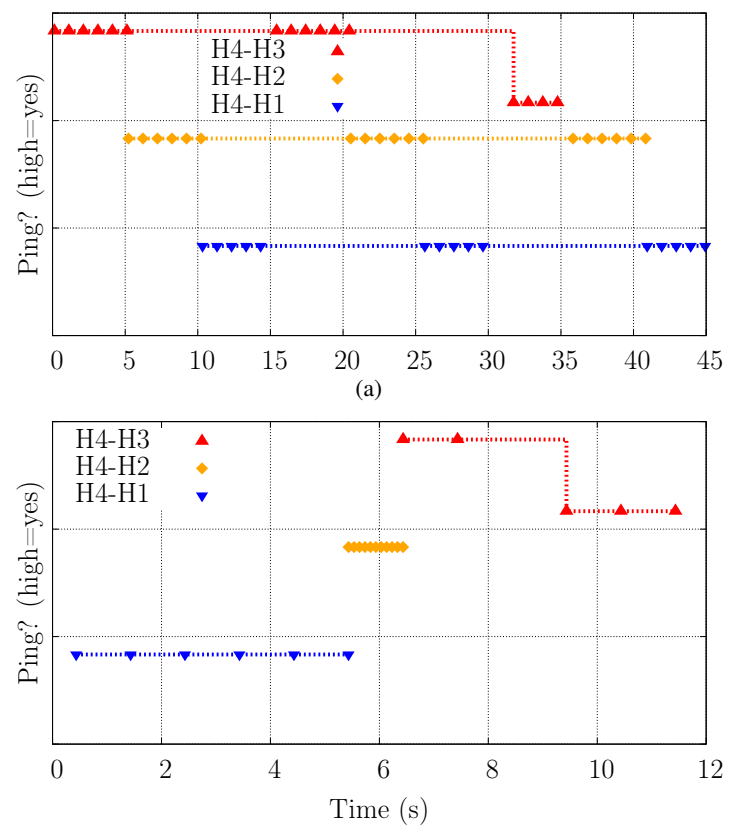

(b)

Figure 15: Intrusion Detection System: (a) correct vs. (b) incorrect.

This IDS example took $0.021 \mathrm{~s}$ to compile and produced 152 flow-table rules. In Figure 15(a), we demonstrate the correct behavior of the program, by first successfully pinging $\mathrm{H} 3, \mathrm{H} 2, \mathrm{H} 1, \mathrm{H} 3, \mathrm{H} 2, \mathrm{H} 1$ (in that order) from $\mathrm{H} 4$. This results in a situation where we have contacted $\mathrm{H} 1$ and then $\mathrm{H} 2$, causing the third attempt to contact $\mathrm{H} 3$ to be blocked (H4-H3 pings dropped). The incorrect (uncoordinated) implementation in Figure 15(b) allows a faulty behavior where we can successfully ping $\mathrm{H} 1$ and then $\mathrm{H} 2$ (in that order), but subsequent $\mathrm{H} 4-\mathrm{H} 3$ traffic is still enabled temporarily.

\subsection{Quantitative Results}

In this experiment, we automatically generated some eventdriven programs which specify that two hosts $\mathrm{H} 1$ and $\mathrm{H} 2$ are connected to opposite sides of a ring of switches. Initially, traffic is forwarded clockwise, but when a specific switch detects a (packet) event, the configuration changes to forward counterclockwise. We increased the "diameter" of the ring (distance from $\mathrm{H} 1$ to $\mathrm{H} 2$ ) up to 8, as shown in Figure 16 , and performed the following two experiments.

1. We used iperf to measure H1-H2 TCP/UDP bandwidth, and compared the performance of our running event-driven program, versus that of the initial (static) configuration of the program running on un-modified OpenFlow 1.0 reference switches/controller. Figure 16(a) shows that our performance (solid line) is very close to the performance of a system which does not do packet tagging, event detection, etc. (dashed line)—we see around $6 \%$ performance degradation on average (note that the solid and dashed lines almost coincide). 


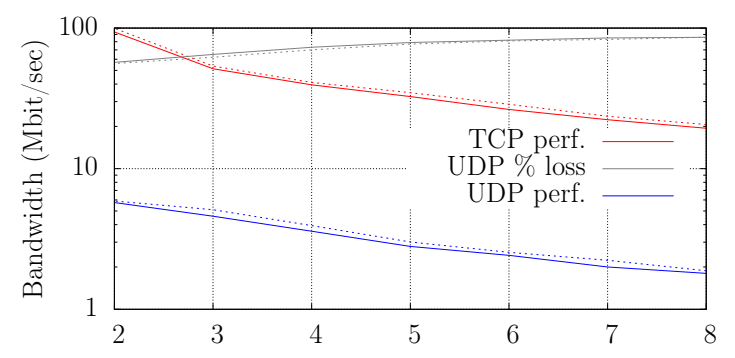

(a)

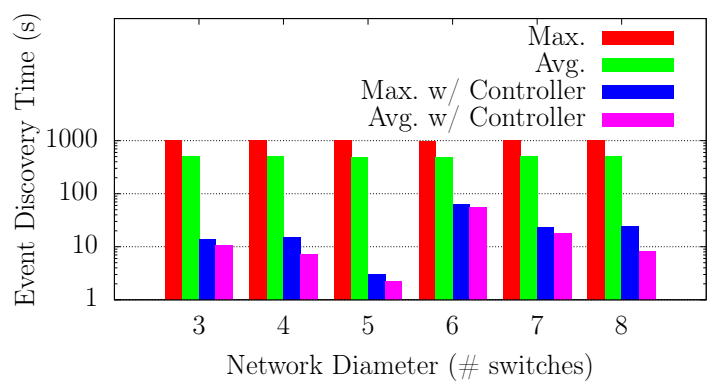

(b)

Figure 16: Circular Example: (a) bandwidth (solid line is ours, dotted line is reference implementation) and (b) convergence.

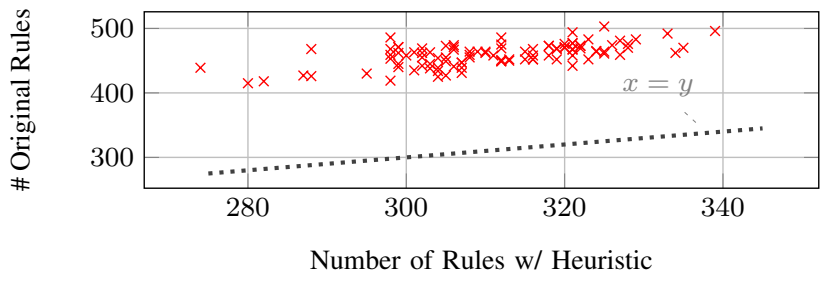

Figure 17: Heuristic: reducing the number of rules.

2. We measured maximum and average time needed for a switch to learn about the event. The "Max." and "Avg." bars in Figure 16(b) are these numbers when the controller does not assist in disseminating events (i.e. only the packet digest is used), and the other columns are the maximum and average when the controller does so.

\subsection{Optimizations}

When a configuration change occurs, the old and new configurations are often similar, differing only in a subset of flowtable rules. Tables are commonly stored in TCAM memory on switches, which is limited/costly, so it is undesirable to store duplicate rules. As mentioned in Section 4.1, each of our rules is guarded by its configuration's numeric ID. If the same rule occurs in several configurations having IDs with the same (binary) high-order bits, intuitively we can reduce space usage by keeping a single copy of the rule, and guarding it with a configuration ID having the shared high-order bits, and wildcarded low-order bits. For example, if rule $r$ is used in two different configurations having IDs 2 (binary 10) and 3 (binary 11), we can wildcard the lowest bit $(1 *)$, and keep a single rule $(1 *) r$ having this wildcarded guard, in-

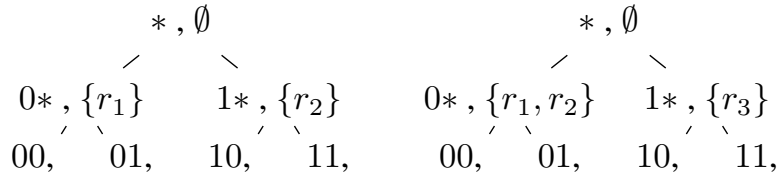

$$
\begin{aligned}
& \left\{r_{1}, r_{2}\right\}\left\{r_{1}, r_{3}\right\}\left\{r_{2}, r_{3}\right\}\left\{r_{1}, r_{2}\right\} \quad\left\{r_{1}, r_{2}\right\}\left\{r_{1}, r_{2}\right\}\left\{r_{1}, r_{3}\right\}\left\{r_{2}, r_{3}\right\}
\end{aligned}
$$

(a)

(b)

Figure 18: Heuristic: two different tries for the same configurations.

stead of two copies of $r$, with the "10" and " 11 " guards. Ideally, we would like to (re)assign numeric IDs to the configurations, such that maximal sharing of this form is achieved.

We formalize the problem as follows. Assume there is a set of all possible rules $\mathcal{R}$. A configuration $C$ is a subset of these rules $C \subseteq \mathcal{R}$. Assume there are $k$ bits in a configuration ID. Without loss of generality we assume there are exactly $2^{k}$ configurations (if there are fewer, we can add dummy configurations, each containing all rules in $\mathcal{R}$ ). For a given set of configurations, we construct a trie having all of the configurations at the leaves. This trie is a complete binary tree in which every node is marked with (1) a wildcarded mask that represents the configuration IDs of its children, and (2) the intersection of the rule-sets of its children.

Consider configurations $C_{0}=\left\{r_{1}, r_{2}\right\}, C_{1}=\left\{r_{1}, r_{3}\right\}$, $C_{2}=\left\{r_{2}, r_{3}\right\}, C_{3}=\left\{r_{1}, r_{2}\right\}$. Figure 18 shows two different assignments of configurations to the leaves of tries. The number of rules for trie (a) is 6: $(0 *) r_{1},(00) r_{2},(01) r_{3}$, $(1 *) r_{2},(10) r_{3},(11) r_{1}$. The number of rules for trie (b) is 5 : $(0 *) r_{1},(0 *) r_{2},(1 *) r_{3},(10) r_{1},(11) r_{2}$. Intuitively, this is because the trie (b) has larger sets in the interior. Our polynomial heuristic follows that basic intuition: it constructs the trie from the leaves up, at each level pairing nodes in a way that maximizes the sum of the cardinalities of their sets. This does not always produce the global maximum rule sharing, but we find that it produces good results in practice.

As indicated by the Figure 17 result (64 randomlygenerate configurations w/ 20 rules), on average, rule savings was about $32 \%$ of the original number of rules. We also ran this on the previously-discussed Firewall, Learning Switch, Authentication, Bandwidth Cap, and IDS examples, and got rule reductions of $18 \rightarrow 16,43 \rightarrow 27,72 \rightarrow 46$, $158 \rightarrow 101$, and $152 \rightarrow 133$ respectively.

\section{Related Work}

Network Updates, Verification, and Synthesis. We already briefly mentioned an early approach known as consistent updates [33]. This work was followed by update techniques that respect other correctness properties [25] [17] [40] [26]. These approaches for expressing and verifying correctness of network updates work in terms of individual packets.

In event-driven network programs, it is necessary to check properties which describe interactions between multiple packets. There are several works which seek to perform 
network updates in the context of multi-packet properties [12] [23]. There are also proposals for synthesizing SDN controller programs from multi-packet examples [39] and from first-order specifications [32]. Lopes et al. presented techniques for verifying reachability in stateful network programs [24], using a variant of Datalog. This is a complimentary approach which could be used as a basis for verifying reachability properties of our stateful programs.

Network Programming Languages. Network programs can often be constructed using high-level languages. The Frenetic project [10] [27] [11] allows higher-level specification of network policies. Other related projects like Merlin [36] and NetKAT [35] [4] provide high-level languages/tools to compile such programs to network configurations. Works such as Maple [37] and FlowLog [31] seek to address the dynamic aspect of network programming.

None of these systems and languages provide both (1) event-based constructs, and (2) strong semantic guarantees about consistency during updates, while our framework enables both. Concurrently with this paper, an approach called SNAP [3] was developed, which enables event-driven programming, and allows the programmer to ensure consistency via an atomic language construct. Their approach offers a more expressive language than our Stateful NetKAT, but in our approach, we enable correct-by-construction eventbased behavior and provide a dynamic correctness property, showing (formally) that is strong enough for easy reasoning, yet flexible enough to enable efficient implementations. We also prove the correctness of our implementation technique.

Routing. The consistency/availability trade-off is of interest in routing outside the SDN context as well. In [18], a solution called consensus routing is presented, based on a notion of causality between triggers (related to our events). However, the solution is different in many aspects, e.g. it allows a transient phase without safety guarantees.

High-Level Network Functionality. Some recent work has proposed building powerful high-level features into the network itself, such as fabrics [9], intents [1], and other virtualization functionality [22]. Pyretic [28] and projects built on top of it such as PyResonance [20], SDX [14], and Kinetic [21] provide high-level operations on which network programs can be built. These projects do not guarantee consistency during updates, and thus could be profitably combined with an approach such as ours.

\section{Discussion and Future Work}

Generality of Our Approach. The event-driven SDN update problem considered in this paper is an instance of a more general distributed-systems programming problem, namely how to write correct and efficient programs for distributed systems. We provide a PL approach (consistency property, programming language, and compiler/runtime) which ensures that the programmer need not reason about interleavings of events and updates for each application, and we show that our consistency model and implementation technique work well in the context of SDN programs, but we do not believe they are limited to that specific arena. Our approach could also possibly be extended to other distributed systems in which availability is prioritized, and consistency can be relaxed in a well-defined way, as in our event-driven consistent updates. Example domains include wireless sensor networks or other message-passing systems where the nodes have basic stateful functionality.

Future Work. There are several directions for future work which could address limitations of our current system.

1. We assume that the set of (potential) hosts is known in advance, and use this information to generate corresponding flow tables for each switch. This may not be the right choice in settings where hosts join/leave. Our approach could be extended to represent hosts symbolically.

2. We currently store all configurations on the switches, so that they are immediately available during updates. Our optimizations allow this to be done in a space-efficient way, but there may be situations when it would be better for the controller to reactively push new configurations to switches. This is an interesting problem due to interleavings of events and controller commands.

3. It would be interesting to consider formal reasoning and automated verification for Stateful NetKAT.

4. We provide a solution to the problem of performing multiple updates, and the dynamic implementations we produce are meant to "run" in the network indefinitely. However, there may be ways to update the running dynamic program itself in some consistent way.

\section{Conclusion}

This paper presents a full framework for correct event-driven programming. Our approach provides a way of rigorously defining correct event-driven behavior without the need for specifying logical formulas. We detail a programming language and compiler which allow the user to write high-level network programs and produce correct and efficient SDN implementations, and we demonstrate the benefits of our approach using real-world examples. This paper considers the challenging problem of distributing an event-based stateful network program, and solves it in a principled way.

\section{Acknowledgments}

Many thanks to the anonymous PLDI reviewers for offering helpful and constructive comments, as well as Zach Tatlock for shepherding our paper and providing useful feedback. Our work is supported by the National Science Foundation under grants CNS-1111698, CNS-1413972, CCF-1421752, CCF-1422046, CCF-1253165, and CCF-1535952; the Office of Naval Research under grant N00014-15-1-2177; and gifts from Cisco, Facebook, Fujitsu, Google, and Intel. 


\section{References}

[1] ONOS Intent Framework. 2014. URL https://wiki. onosproject.org/x/XgAz.

[2] C. J. Anderson, N. Foster, A. Guha, J.-B. Jeannin, D. Kozen, C. Schlesinger, and D. Walker. NetKAT: Semantic Foundations for Networks. POPL, 2014.

[3] M. T. Arashloo, Y. Koral, M. Greenberg, J. Rexford, and D. Walker. SNAP: Stateful Network-Wide Abstractions for Packet Processing. 2015.

[4] R. Beckett, M. Greenberg, and D. Walker. Temporal NetKAT. PLVNET, 2015.

[5] G. Bianchi, M. Bonola, A. Capone, and C. Cascone. OpenState: Programming Platform-independent Stateful Openflow Applications Inside the Switch. ACM SIGCOMM CCR, 2014.

[6] P. Bosshart, D. Daly, G. Gibb, M. Izzard, N. McKeown, J. Rexford, C. Schlesinger, D. Talayco, A. Vahdat, G. Varghese, et al. P4: Programming Protocol-independent Packet Processors. ACM SIGCOMM CCR, 2014.

[7] E. Brewer. Towards robust distributed systems (abstract). $P O D C$, page 7, 2000.

[8] M. Casado, M. J. Freedman, J. Pettit, J. Luo, N. McKeown, and S. Shenker. Ethane: Taking Control of the Enterprise. SIGCOMM, 2007.

[9] M. Casado, T. Koponen, S. Shenker, and A. Tootoonchian. Fabric: A Retrospective on Evolving SDN. HotSDN, 2012.

[10] N. Foster, R. Harrison, M. J. Freedman, C. Monsanto, J. Rexford, A. Story, and D. Walker. Frenetic: A Network Programming Language. ICFP, 2011.

[11] N. Foster, A. Guha, M. Reitblatt, A. Story, M. J. Freedman, N. P. Katta, C. Monsanto, J. Reich, J. Rexford, C. Schlesinger, et al. Languages for Software-Defined Networks. Communications Magazine, IEEE, 51(2):128-134, 2013.

[12] S. Ghorbani and B. Godfrey. Towards Correct Network Virtualization. HotSDN, 2014

[13] S. Gilbert and N. Lynch. Perspectives on the CAP Theorem. IEEE Computer, 45(2):30-36, 2012.

[14] A. Gupta, L. Vanbever, M. Shahbaz, S. P. Donovan, B. Schlinker, N. Feamster, J. Rexford, S. Shenker, R. Clark, and E. Katz-Bassett. SDX: A Software Defined Internet Exchange. SIGCOMM, 2014.

[15] C.-Y. Hong, S. Kandula, R. Mahajan, M. Zhang, V. Gill, M. Nanduri, and R. Wattenhofer. Achieving High Utilization with Software-driven WAN. SIGCOMM, 2013.

[16] S. Jain et al. B4: Experience with a Globally-Deployed Software Defined WAN. SIGCOMM, 2013.

[17] X. Jin, H. H. Liu, R. Gandhi, S. Kandula, R. Mahajan, M. Zhang, J. Rexford, and R. Wattenhofer. Dynamic Scheduling of Network Updates. SIGCOMM, 2014.

[18] J. John, E. Katz-Bassett, A. Krishnamurthy, T. Anderson, and A. Venkataramani. Consensus Routing: The Internet as a Distributed System. NSDI, 2008.

[19] N. Kang, Z. Liu, J. Rexford, and D. Walker. Optimizing the One Big Switch Abstraction in Software-Defined Networks. CoNEXT, 2013.
[20] H. Kim, A. Gupta, M. Shahbaz, J. Reich, N. Feamster, and R. Clark. Simpler Network Configuration with State-Based Network Policies. Technical report, Georgia Tech, 2013.

[21] H. Kim, J. Reich, A. Gupta, M. Shahbaz, N. Feamster, and R. Clark. Kinetic: Verifiable Dynamic Network Control. NSDI, 2015.

[22] T. Koponen, K. Amidon, P. Balland, M. Casado, A. Chanda, B. Fulton, I. Ganichev, J. Gross, N. Gude, P. Ingram, et al. Network Virtualization in Multi-tenant Datacenters. NSDI'14.

[23] W. Liu, R. B. Bobba, S. Mohan, and R. H. Campbell. InterFlow Consistency: Novel SDN Update Abstraction for Supporting Inter-Flow Constraints. NDSS, 2015.

[24] N. P. Lopes, N. Bjørner, P. Godefroid, K. Jayaraman, and G. Varghese. Checking Beliefs in Dynamic Networks. NSDI, 2015.

[25] A. Ludwig, M. Rost, D. Foucard, and S. Schmid. Good Network Updates for Bad Packets: Waypoint Enforcement Beyond Destination-based Routing Policies. HotNets, 2014.

[26] J. McClurg, H. Hojjat, P. Cerny, and N. Foster. Efficient Synthesis of Network Updates. PLDI, 2015.

[27] C. Monsanto, N. Foster, R. Harrison, and D. Walker. A Compiler and Run-time System for Network Programming Languages. POPL, 2012.

[28] C. Monsanto, J. Reich, N. Foster, J. Rexford, and D. Walker. Composing Software Defined Networks. NSDI, 2013.

[29] M. Moshref, M. Yu, A. B. Sharma, and R. Govindan. Scalable Rule Management for Data Centers. NSDI, 2013.

[30] M. Moshref, A. Bhargava, A. Gupta, M. Yu, and R. Govindan. Flow-level State Transition as a New Switch Primitive for SDN. 2014.

[31] T. Nelson, A. D. Ferguson, M. Scheer, and S. Krishnamurthi. Tierless Programming and Reasoning for Software-Defined Networks. NSDI, 2014.

[32] O. Padon, N. Immerman, A. Karbyshev, O. Lahav, M. Sagiv, and S. Shoham. Decentralizing SDN Policies. POPL, 2015.

[33] M. Reitblatt, N. Foster, J. Rexford, C. Schlesinger, and D. Walker. Abstractions for Network Update. SIGCOMM'12.

[34] A. Sivaraman, M. Budiu, A. Cheung, C. Kim, S. Licking, G. Varghese, H. Balakrishnan, M. Alizadeh, and N. McKeown. Packet Transactions: A Programming Model for DataPlane Algorithms at Hardware Speed. 2015.

[35] S. Smolka, S. Eliopoulos, N. Foster, and A. Guha. A Fast Compiler for NetKAT. ICFP, 2015.

[36] R. Soulé, S. Basu, P. J. Marandi, F. Pedone, R. Kleinberg, E. G. Sirer, and N. Foster. Merlin: A Language for Provisioning Network Resources. CoNEXT, 2014.

[37] A. Voellmy, J. Wang, Y. R. Yang, B. Ford, and P. Hudak. Maple: Simplifying SDN Programming Using Algorithmic Policies. SIGCOMM, 2013.

[38] G. Winskel. Event Structures. Springer, 1987.

[39] Y. Yuan, D. Lin, R. Alur, and B. T. Loo. Scenario-based Programming for SDN Policies. CoNEXT, 2015.

[40] W. Zhou, D. Jin, J. Croft, M. Caesar, and P. B. Godfrey. Enforcing Generalized Consistency Properties in SoftwareDefined Networks. NSDI, 2015. 